

\title{
THE IMMUNE CHECKPOINT CD96 DEFINES A DISTINCT LYMPHOCYTE PHENOTYPE AND IS HIGHLY EXPRESSED ON TUMOR-INFILTRATING T CELLS
}

\author{
Ailin Lepletier ${ }^{1}$, Viviana P Lutzky ${ }^{1}$, Deepak Mittal ${ }^{1}$, Kimberley Stannard ${ }^{1}$, \\ Thomas S Watkins ${ }^{1,2}$, Champa N Ratnatunga ${ }^{1,2,3}$, Corey Smith ${ }^{1}$, Helen M McGuire ${ }^{4,5}$, \\ Roslyn A Kemp ${ }^{6}$, Pamela Mukhopadhyay ${ }^{1}$, Nicola Waddell ${ }^{1}$, Mark J Smyth ${ }^{1}$, \\ William C Dougall ${ }^{1}$, John J Miles ${ }^{1,2,3,7,8}$
}

\footnotetext{
1. QIMR Berghofer Medical Research Institute, Brisbane, QLD 4006, Australia.

2. Faculty of Medicine, The University of Queensland, Brisbane, QLD 4029, Australia.

3. Centre for Biodiscovery and Molecular Development of Therapeutics, AITHM, James Cook University, Cairns, QLD 4870, Australia.

4. Discipline of Pathology, School of Medical Sciences, Faculty of Medicine and Health, University of Sydney, Sydney, NSW 2006, Australia.

5. Ramaciotti Facility for Human Systems Biology, University of Sydney, Sydney, NSW 2006, Australia

6. Department of Microbiology and Immunology, University of Otago, Dunedin 9010, New Zealand.

7. Division of Infection and Immunity, Cardiff University School of Medicine, Cardiff CF14 4XN, UK.

8. Medicine, Dentistry and Health, Griffith University, Brisbane, QLD 4111, Australia.
}

\begin{abstract}
CD96 has recently been shown to be a potent immune checkpoint molecule in mice, but a similar role in humans is not known. In this study, we provide a detailed map of CD96 expression across human lymphocyte lineages, the kinetics of CD96 regulation on T-cell activation and co-expression with other conventional and emerging immune checkpoint molecules. We show that CD96 is predominantly expressed by T cells and has a unique lymphocyte expression profile. CD96 high $T$ cells exhibited distinct effector functions on activation. Of note, CD96 expression was highly correlated with T-cell markers in primary and metastatic human tumors and was elevated on antigen-experienced T cells and tumorinfiltrating lymphocytes. Collectively, these data demonstrate that CD96 may be a promising immune checkpoint to enhance T-cell function against human cancer and infectious disease.
\end{abstract}

\section{INTRODUCTION}

Endogenous antitumor immunity is limited by the immunosuppressive tumor microenvironment. However, reactivation of immune responses and overcoming immune tolerance have been achieved with antibody blockade of T-cell co-inhibitory receptors CTLA4 and PD-1 or the immunosuppressive ligand PD-L1. While impressive overall response rates have been observed in cancer patients treated with these immune-therapies, combination treatment in patients with advanced malignant melanoma has produced even more significant anticancer effects. ${ }^{1,2}$ Despite these impressive clinical responses, some patients do not respond to these therapies or do not demonstrate durable responses, suggesting that alternative immunosuppressive mechanisms must be targeted in combination to provide maximal therapeutic benefit across all individuals.

Emerging data have demonstrated that members of the immunoglobulin (Ig)-like receptor family, CD96, CD226 (DNAM-1) and TIGIT, modulate NK and T-cell activity in cancers and fine-tune tumor immunosurveillance. ${ }^{3}$ These receptors interact with multiple cognate nectin and nectin-like protein ligands, including CD155 and CD112, present in cancer cells, and reactive myeloid and antigen-presenting cells (APC) within the tumor environment. The recognition of CD155 by CD226 is critical for antitumor immunity as demonstrated by the 
accelerated growth and metastasis of chemically or genetically induced tumors in CD226 ${ }^{-/-}$ mice. $^{4,5}$

In mouse tumor models, anti-TIGIT monoclonal antibodies (mAbs) have demonstrated profound antitumor efficacy when used in combination with anti-PD-L1 or anti-PD1, and this mechanism is thought to involve the inhibitory function of TIGIT on regulatory T cells (Tregs) ${ }^{6}$ and $\mathrm{CD}^{+}{ }^{+} \mathrm{T}_{\text {cells }}{ }^{7}$; however, detailed pathway information is lacking. An additional role for TIGIT on NK-cell-mediated control of metastasis has also been defined ${ }^{8,}$ 9; but again, detailed pathway information is lacking ${ }^{6}$. In human melanoma tumor-infiltrating lymphocytes (TILs), anti-TIGIT mAbs augmented tumor antigen-specific T-cell degranulation, cytokine production and proliferation have been observed ${ }^{10}$. An intrinsic inhibitory function of CD96 was first shown using CD96-deficient mice, where NK cells produced greater IFNy in response to lipopolysaccharide (LPS), IL12 or IL18 stimulation ${ }^{8}$. A profound role for CD96 in resistance to spontaneous or experimental lung metastases and MCA-induced fibrosarcomas was demonstrated in CD96-deficient mice or on blockade using CD96specific mAbs in wild-type mice ${ }^{8,11}$. Mechanistically, the function of CD96 was dependent on NK cells, IFNy and CD226, while an intrinsic function of CD96 on T cells was not addressed. It is therefore hypothesized that CD226 provides an activation signal to NK and T cells while TIGIT and CD96 may provide "inhibitory" signals thereby counterbalancing CD226 signaling. In the present study, we have expanded the existing experimental evidence suggesting an inhibitory function of mouse CD96 on immune-mediated tumor control and provide a comprehensive expression analysis of CD96 in human immune cell subsets and in primary and metastatic tumors.

\section{RESULTS}

\section{CD96 expression on lymphocytes is compartmentalized and unique}

It was recently shown that modulation of the CD96 pathway enhanced antitumor responses in mice ${ }^{11}$. Whether these data can be translated to human cancer patients is unknown. Given the limited knowledge of CD96 expression profiles in humans, our first aim was to map CD96 expression across human lymphocyte subsets ex vivo and contrast expression with other immune checkpoints. Using five high-dimensional flow cytometry panels, we performed a comprehensive dissection of CD96 expression using clone NK92.39 in the peripheral blood of healthy adults (Figure 1). Across the major lymphocyte lineages, T cells had the highest percentage of $\mathrm{CD}^{+} 6^{+}$cells $(38 \pm 9 \%)$ followed by NKCD56-bright cells $(21 \pm 8 \%)$ (Figure 1a). Little or no CD96 was observed in B cells or monocytes. Interestingly, the overall CD96 expression hierarchy differed from the immune checkpoints TIGIT, CD226 and PD-1 (Figure 1a). Here, TIGIT expression was greatest in CD56 ${ }^{+}$T cells (48 $\pm 8 \%$ ), followed by NKCD56-dim (36 $\pm 15 \%)$. CD226 was highest in NKCD56-bright cells $(97.53 \pm 1 \%)$ and PD-1 expression was highest in T cells (mean $7 \pm 2 \%$ ). We next determined CD96 expression on T cells by subtype and found CD96 was highest in mucosal-associated invariant T (MAIT) cells (48 $\pm 18 \%$ ) and CD8 ${ }^{+}$T cells (46 $\pm 12 \%$ ) (Figure 1b). A notable deficiency of CD96 was observed in $\gamma \delta$ T cells and Tregs while TIGIT expression was enriched in these subsets. We next examined CD96 expression by T-cell phenotype across CD4+ and CD8+ ${ }^{+}$-cell lineages covering naïve, central memory, effector memory (EM) and effector memory RA (Figure 1c). In general, CD96 expression was higher in CD8 ${ }^{+}$T-cell lineages, with EM (57 $\left.\pm 16 \%\right)$ and central memory cells $(42 \pm 15 \%)$ showing the highest percentage of CD96 ${ }^{+}$cells.

In CD4 ${ }^{+} \mathrm{T}$ cells, CD96 expression was also highest in the EM phenotype (52 $\left.\pm 12 \%\right)$. Across T-cell phenotypes, the hierarchical pattern of CD96 expression again differed from the $\mathrm{Ig}$ 
superfamily inhibitory receptor TIGIT but was similar to CD226 and PD-1. Within CD4+ Thelper subtypes (Th1, Th2 and Th17) CD96 expression was highest on Th1 cells (15 $\pm 4 \%)$ with PD-1 being the closest hierarchical match to CD96 (Figure 1d). Across all flow cytometric data, the relative differences in percent of $\mathrm{CD}^{2} 6^{+}$cells were matched by relative differences in mean fluorescence intensity (data not shown). This correlation between percentage expression and mean fluorescence intensity was also seen with PD-1, TIGIT and CD226 (data not shown). Collectively, these data highlight selective CD96 distribution across human immune subsets and a generally distinct pattern compared with the related inhibitory receptor TIGIT, which correlated predominantly with regulatory cells (Treg and Th2).

\section{CD96 shows co-expression bias with other checkpoint molecules}

The degree of co-expression of other receptors with CD96 may provide insight into CD96 functionality, especially given the potential to counterbalance CD226 action or to regulate other inhibitory receptor signaling. In resting $\mathrm{CD}^{+} \mathrm{T}$ cells, co-expression of CD96 with CD226 was greater $(29 \pm 13 \%)$ than TIGIT (11\% $\pm 5 \%)$ and PD-1 (3 $\pm 2 \%)$ (data not shown). When gating on resting $\mathrm{CD} 96^{\text {high }} \mathrm{CD} 4^{+} \mathrm{T}$ cells, the co-expressing hierarchy was identical, comprising CD226 (35 $\pm 9 \%)$ followed by TIGIT (7 $\pm 3 \%$ ) and PD-1 (4 $\pm 1 \%$ ) (data not shown). PD-1 expression was generally low, which is consistent with a low level of antigen receptor stimulation in PBMC. Using resting or activated PBMC, we next concatenated the multiparametric flow data and performed viSNE analysis which allows visualization of highdimensional single-cell data. When examining resting CD8 ${ }^{+}$T-cell subsets, we observed generally heterogeneous CD96 expression and the greatest overlap with CD226 expression and a relative enrichment of CD96 in the EM population (CD45RA ${ }^{-}$and $C C R 7^{-}$) (Figure 2a). CD96 was observed to be co-expressed in resting PBMCs, with examples of CD96/TIGIT double-positive cells observed in both PD-1 positive and PD-1 negative CD8 ${ }^{+}$T-cell subsets. When examining resting CD4+ T-cell subsets, we observed CD96 to cluster across the

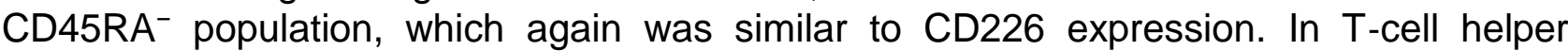
subsets, both CD96 and CD226 expression were heterogeneous across Th1, Th2 and Th17 cells (Figure $2 b$ ), although there was slightly lower expression of CD96 in Th2 cells. Conversely, PD-1 and TIGIT expression clustered in a population of cells producing both IFNy and IL17 from phorbol myristate acetate/ionomycin-activated T cells.

\section{CD96 ${ }^{\text {high }} \mathrm{T}$ cells have a unique resting and activated transcription landscapes}

Given the enrichment of CD96 in certain T-cell subsets, we next examined whether CD96 expression correlated with unique cell phenotype and functional phenotype. To determine this, we sorted CD96 ${ }^{\text {high }}$ and CD96 ${ }^{\text {low }}$ cells across CD4 ${ }^{+}$and CD8 ${ }^{+}$memory T cells from 10 healthy adults to high purity and quantified T-cell transcriptional signatures. Through analysis of multidimensional immunological datasets ${ }^{12}$, we found that CD96 ${ }^{\text {high }}$ and CD96 low phenotypes exhibit unique transcriptional landscapes across both memory CD4 ${ }^{+}$and $\mathrm{CD}^{+}$ lineages. From transcriptional signatures across cohorts, canonical correspondence analysis (CCA) showed $\mathrm{CD}^{+}$memory had higher variation $(P=0.007)$ compared with $\mathrm{CD}^{+}$ memory $(P=0.04)$ (Figure 3a, b). Compared with paired CD96 ${ }^{\text {low }}$ sorts, CD96 ${ }^{\text {high }} \mathrm{CD}^{+}$and $\mathrm{CD}^{+}$cells showed 53 and 46 differentially expressed genes, respectively. Of note, CD96 RNA levels aligned with CD96 ${ }^{\text {high }}$ and CD96 low cells which validated sort purity (Supplementary tables 3 and 4). Paired $t$-test analysis of significantly expressed genes can be found in the supplementary material (Supplementary tables 3 and 4). Analysis using either CAA or support vector machine trained on the transcriptional profiles of either CD96 ${ }^{\text {high }}$ 
or CD96 ${ }^{\text {low }}$ T-cells data suggests CD96-associated phenotype is stronger in the CD8+ ${ }^{+}$-cell compartment.

For biological relevance, we next validated some of the top mRNA hits using multiparametric flow cytometry (Supplementary Figure 1a, b) on antigen-specific CD8 ${ }^{+} \mathrm{T}$ cells using tetramers and relevant functional markers (Supplementary tables 1 and 3) across four donors. For GZMB and TBX21 (both within the top four most significant genes), we observed significant differences between CD96 ${ }^{\text {high }}$ and CD96 ${ }^{\text {low }}$ T-cell populations that matched the direction seen in the NanoString profiling (Supplementary table 3).

To identify possible differences in function between phenotypes, we sorted CD96 high and $\mathrm{CD}^{\text {low }}$ memory $\mathrm{CD}^{+} \mathrm{T}$ cells from five donors and then activated each population with phorbol myristate acetate/ionomycin. Transcript analysis indicated differentially upregulated or downregulated signatures between CD96 ${ }^{\text {high }}$ and CD96low memory CD8 ${ }^{+}$T-cells postactivation. Using CCA, we determined that activation significantly changed the CD96 ${ }^{\text {high }}$ $(P=0.01)$ and $\mathrm{CD} 6^{\text {low }}(P=0.01)$ populations when comparing to a resting state. Using paired $t$-tests on the fold change for each donor, we identified 14 genes that were differentially expressed between CD96 ${ }^{\text {high }}$ and CD96 low $T$ cells following activation (Supplementary table 4). CCA was not statistically significant between populations and, compared with ex vivo profiling, fewer genes were differentially expressed between CD96 ${ }^{\text {high }}$ and CD96 low T-cells postactivation, suggesting that the populations become more homogeneous after a strong activation signal. We have previously observed this "blending" phenotype when examining $\mathrm{CD}^{+}$and $\mathrm{CD}^{+}$human $\mathrm{T}$-cell lineages post-T cell receptor (TCR) activation ${ }^{12}$. Nonetheless, important functional genes were differentially expressed during activation including a strong downregulation of the protein tyrosine phosphatase CD45RA in CD96 ${ }^{\text {high }}$ cells $(P=0.0005)$ and upregulation of the pleotropic cytokine IL23A $(P=0.02)$ (Figure 3c). Likewise, activated CD96 low $\mathrm{T}$ cells showed strong downregulation of the effector molecule granzyme M (GZMM) $(P=0.012)$ and immune checkpoint CTLA-4 $(P=0.037)$ postactivation (Figure 3c). Significant differences were also observed in effector molecules (GZMB) cytokines/chemokines (IL2, IL17A, IL22, IFNy and MIP-1 $\beta$ ), integrins (ILTGA4), transcription factors (JUNB), cytolytic markers (CD160), the adenosine pathway (NT5E) and the JAK-STAT pathway (JAK, STAT5B and STAT6) (Supplementary table 4). Overall, these data indicate that CD96 ${ }^{\text {high }} \mathrm{T}$ cells have a distinctive ex vivo phenotype and exhibit a distinct functional phenotype when activated.

\section{CD96 is markedly upregulated on human T-cells poststimulation}

We next examined CD96 expression kinetics and co-expression with other immune checkpoint receptors in $\mathrm{T}$ cells after stimulation with CD3/CD28 microbeads. CD96 expression (mean fluorescence intensity) on $\mathrm{CD}^{+} \mathrm{T}$ cells decreased at $4 \mathrm{~h}$ postactivation and remained low at D1 relative to ex vivo resting cells (Figure 4a). CD96 expression increased at D3 relative to the nadir between $4 \mathrm{~h}$ and D1, with almost all CD3 ${ }^{+} \mathrm{T}$-cells expressing CD96 at D5 (89 $\pm 9 \%$ ) and persistently high expression to D7 (Figure 4a, b). The expression profile kinetics for CD226 was most closely matched to CD96, as CD226 expression was reduced at D1 relative to ex vivo resting cells, followed by increased expression at D5 that persisted to D7 (Figure 4a, b). Upon activation, both TIGIT and PD-1 also demonstrated increased expression relative to ex vivo samples; however, overall the magnitude fold increase was substantially greater ( 8-fold) for CD96 compared with other markers. Analysis of mRNA for each of these immune receptors revealed TIGIT upregulation at $4 \mathrm{~h}$ postactivation, followed by PD-1 and CD226 upregulation at D1, while any increase in CD96 was not observed until D3 (Figure 4c). A comparative analysis of CD96 protein 
expression kinetics (Figure 4a, b) with expression of CD96 mRNA (Figure 4c) suggested that protein expression was regulated as result of changes in the mRNA encoding CD96 isoform 1, as CD96 isoform 2 mRNA remained low during the 7 days of T-cell stimulation. Analysis of T cells at D7 poststimulation demonstrated an enhanced co-expression of CD96 with CD226 or TIGIT in both $\mathrm{CD}^{+}$and $\mathrm{CD}^{+} \mathrm{T}$ cells (Figure $5 \mathrm{a}$ and Supplementary Figure 2a). An increased co-expression of CD96 with PD-1 was also observed in CD4 ${ }^{+} \mathrm{T}$ cells (Supplementary Figure 2a).

Given this understanding of receptor expression dynamic upon broad TCR activation, we next investigated the co-expression of CD96 with other immune receptors in two physiologically relevant systems. First, we determined CD96 expression was upregulated on CD4+ $\mathrm{T}$ cells in an allo-mixed lymphocyte reaction after 5 days of culture (Supplementary Figure 2) while, reciprocally, the ligands CD112 and CD155 were upregulated on LPSactivated/matured dendritic cell (Supplementary Figure 2c). Second, we determined CD96 expression was upregulated on peripheral blood-derived $\mathrm{CD}^{+}$and $\mathrm{CD}^{+} \mathrm{T}$ cells from Epstein-Barr virus-positive donors stimulated in a memory recall assay with their autologous virus-transformed LCL (Supplemental Figure 3a). Here, past experience showed that $\sim 15 \%$ of $T$ cells are antigen specific at day 7 of activation. Similar to the observations with CD3/CD28 stimulated T cells, we observed the greatest magnitude increases for CD96 and CD226 expression while marginal increases in TIGIT and PD-1 were noted. Overall, there was an upregulation of CD96, TIGIT and CD226 expression within CD8 ${ }^{+}$and $\mathrm{CD}^{+}$and a significant increase in CD8 ${ }^{+}$T-cells co-expressing CD96 and PD-1, CD96 and TIGIT or CD96 and CD226 (Figure 5b and Supplementary Figure $3 a$ and b). In order to discriminate whether CD96 expression was associated with hyporesponsiveness as opposed to terminal differentiation, we analyzed co-expression with TIGIT, CD226 or PD-1 within proliferating (CellTrace Violet) $\left(\mathrm{CTV}^{-}\right)$versus nonproliferating $\left(\mathrm{CTV}^{+}\right)$populations after 7 days of stimulation with autologous LCL cells. Comparison of $\mathrm{CTV}^{+}$with $\mathrm{CTV}^{-} \mathrm{CD}^{+} \mathrm{T}$ cells indicated a striking enrichment in CD96/TIGIT and CD96/CD226 double-positive cells in the proliferative subset (Figure 5b). CD155 expression was observed on the LCL cell lines, although high variability ( $<0.5 \%$ to $47 \%$ ) was observed between donors. CD155 was also variably expressed and upregulated on $<10 \%$ of $\mathrm{CD}^{+}{ }^{+}$and $\mathrm{CD}^{+} \mathrm{T}$ cells during the course of the assay (data not shown).

\section{CD96 is highly expressed in human tumors and correlates with antigen-exposure and CD8+ T-cell infiltration}

Given the observed focused expression and activation-dependent upregulation of CD96 on human $\mathrm{CD}^{+}$and $\mathrm{CD}^{+} \mathrm{T}$ cells, we next analyzed the correlation between CD96 and T-cell markers using mRNA expression data for primary and metastatic human melanoma (SKCM), breast invasive carcinoma (BRCA) and uterine corpus endometroid carcinoma from the Cancer Genome Atlas (TCGA). CD96 expression was highly correlated with CD3E, CD4 and CD8A in SKCM with similar CD96 levels observed in metastatic melanoma compared with primary tumors (Figure 6a). The correlation of CD96 mRNA with T-cell markers was highly concordant across primary and metastatic melanoma [Spearman's correlation coefficient $(\rho)=0.88$ and $0.89,0.67$ and 0.77 , and 0.89 and 0.86 , for CD3E, CD4 and CD8A correlations between primary and metastatic melanoma, respectively] (Figure 6a). Notably, the correlation of CD96 and CD8A was higher than that observed for CD96 with CD4, although both associations were relatively high. Similarly, the correlation of CD96 and T-cell markers was also high in BRCA and uterine corpus endometroid carcinoma, again with a relatively higher correlation with CD8A $(P=0.88$ and 0.78$)$ 
compared with CD4 $(P=0.74$ and 0.68$)$ for BRCA and uterine corpus endometroid carcinoma, respectively (Supplementary Figure 4). Analysis of each of the tumor types available from TCGA indicated that the correlation of CD96 with T-cell markers was consistently observed, with the correlation with CD8A being particularly strong (24/31 tumor types demonstrating a Spearman's correlation coefficient $>0.75$ ) (Figure 6b). The high degree of correlation between CD96 and T-cell markers was very similar to that observed with a prototypical Ig supefamily member, TIGIT, and T-cell markers (Figure 6a, b and Figure 3). Collectively, the expression of CD96 and CD8A are higher when comparing metastatic tumor versus primary (Figure 6a) and between normal tissue and tumor (Supplementary Figure 4).

CD96 expression and function has also been previously described on NK cells and a moderate correlation between CD96 and NCR1 was observed in SKCM, uterine corpus endometroid carcinoma and BRCA $(P=0.68, P=0.52$ and $P=0.69$, respectively) (Figure 6a, b, Supplementary Figure 4). As with correlations between CD96 and T-cell markers, the relative correlation of CD96 to a NK marker was similar to that observed for TIGIT and broadly observed across multiple tumor types (14/31 tumor types with Spearman's coefficient $>0.5$ ) (Figure 6b). In contrast to the concordant associations with Tcell markers between primary and metastatic melanoma, the ratio of either CD96 or TIGIT with NCR1 was significantly higher in metastatic versus primary lesions $(P<0.005$ for both genes, Figure 6a).

We next sought to determine whether CD96 was expressed on tetramer ${ }^{+} \mathrm{CD}^{+} \mathrm{T}$ cells and CD8 ${ }^{+}$TILs. Using seven different herpesvirus-specific tetramers on 12 samples, we found an average of $3.73 \%( \pm 6 \%)$ of $\mathrm{CD}^{+} \mathrm{T}^{+}$cells to be tetramer ${ }^{+}$and $26.7 \%( \pm 15.0 \%)$ of tetramer ${ }^{+}$ cells to be $\mathrm{CD}^{+} 6^{+}$(Supplementary table 1). Using CyTOF analysis and the CD96 mAb clone 6F9 (instead of NK92.39), we next analyzed CD96 expression on CD3 ${ }^{+} \mathrm{T}$ cells in the PBMC and TILs of 13 colorectal cancer patients (Figure 6c). We found the frequency of CD96 ${ }^{+} \mathrm{T}$ cells was significant increased $(57 \% \pm 38 \%)(P=<0.0001)$ on TILs compared to those in peripheral blood. These CyTOF data and TCGA data suggest CD96 may be a druggable target for immune modulation in humans.

\section{DISCUSSION}

Immunotherapies that target the checkpoint inhibitory pathways PD-1/PD-L $11^{3}$ or CTLA-4 ${ }^{14}$ have demonstrated profound clinical benefit in cancer patients; however, there is only a subset of patients that experience durable responses upon monotherapy treatment. Combination immunotherapy targeting anti-CTLA4 together with anti-PD-1 produced superior tumor responses and survival benefit in advanced melanoma, and demonstrates the importance of identifying and targeting nonredundant mechanisms of immune evasion by tumors ${ }^{15}$. One feasible approach is to identify and block additional inhibitory receptors on hyporesponsive CD8 ${ }^{+} \mathrm{T}$ cells to augment antitumor function. Additional inhibitory receptors such as TIM-3, LAG-3, BTLA, Vista and B7-H4 have been identified on chronically stimulated $T$ cells and antibodies that block the respective ligands suggest these pathways contribute to $\mathrm{CD}^{+}$T-cell dysfunction in functionally independent manners ${ }^{16-19}$. Recent preclinical studies have identified CD96 and TIGIT as co-inhibitory Ig superfamily receptors which function on lymphocytes to counterbalance the costimulatory CD226. CD226 plays a dominant and central role in NK and T-cell-mediated antitumor immunity ${ }^{3,20}$ and recent murine work supports an inhibitory role for CD96 21 . The functional interplay between the activating CD226 and the inhibitory CD96 and TIGIT receptors is reminiscent of the CTLA$4 / C D 28$ counterbalance that is critical for the fine tuning of the immune response to cancer ${ }^{22}$. 
Demonstration of TIGIT expression and function in infiltrating T cells from human tumors ${ }^{10}$ has provided evidence for the translational potential for this pathway in cancer treatment; however, similar expressional validation of CD96 in human immune cells has limited any translational progression of this target.

In the present study, we provide a comprehensive expression analysis for CD96 across human immune mononuclear cell subsets and in the context of functional CD4 ${ }^{+}$and $\mathrm{CD} 8^{+}$ T-cell responses, including antitumor immunity. In PBMC from healthy donors, CD96 is predominantly expressed by $\mathrm{CD} 8^{+} \mathrm{T}$ cells and, across $\mathrm{T}$-cell subtypes, the pattern of expression is distinct from TIGIT and is generally most closely related to CD226 and PD-1. Within CD4 ${ }^{+} \mathrm{T}$ cells, CD96 was more selectively expressed in Th1 cells compared with TIGIT, which was more highly expressed in Th2 and Treg cells. CD96 and CD226 were more highly expressed in CD8 ${ }^{+}$EM populations as compared with PD-1 and TIGIT; however, PD-1-matched CD96 as the predominant marker for $\mathrm{CD}^{+}$and $\mathrm{CD} 8^{+} \mathrm{EM}$ populations. NanoString transcriptomic profiling across both $\mathrm{CD} 4^{+}$and $\mathrm{CD} 8^{+} \mathrm{T}$ cells determined that CD96 expression delineates a distinct T-cell phenotype and distinct function, suggesting a relationship between the CD96 pathway and differentiation status and/or activation potential in vivo.

The kinetics of CD96 expression in T cells upon TCR stimulation was distinct from the other inhibitory receptors TIGIT and PD-1 and the "trough-then-peak" expression kinetics of CD96 was similar to that observed for CD226.

CD226 plays a central role in stimulating T-cell and NK-cell antitumor immunity and, at the same time, contributes to the limiting, immunomodulatory effects of TIGIT and CD96 ${ }^{7,8}$. Each of these receptors binds to CD155, which is expressed by many solid and hematological malignancies; high CD155 expression has been reported to correlate with unfavorable outcomes in patients with melanoma ${ }^{23}$, lung adenocarcinoma ${ }^{24}$ or colorectal carcinoma ${ }^{25}$. Significantly, while CD155 expression on tumors can be regulated through therapeutic intervention or by genotoxic or oxidative stress ${ }^{26}$, the additional expression of CD155 on reactive myeloid cells infiltrating human tumors is particularly prominent, exceeding levels observed for PD-L1 ${ }^{10,27}$. Competition of CD155 or CD112 ligand binding to inhibitory versus activating receptors can influence the net activation signal in lymphocytes ${ }^{23}$, although the potential for receptor/receptor homo- and heterodimerization between TIGIT, CD226 and CD96 may also contribute to the degree of activation. CD226 expression is lower in chronically stimulated NK cells in a mouse multiple myeloma mode ${ }^{28}$ and $\mathrm{CD}^{+}$TILs from melanoma patients ${ }^{10}$, suggesting that a lower ratio of activating:inhibitory receptors will limit lymphocyte responses. In the present study, we observed that the kinetics and pattern of CD96 expression was similar to CD226 upon Tcell activation and, importantly, CD96 and CD226 were highly co-expressed on T cells. The distinct kinetics and expression patterns of CD96 and TIGIT on T cells defined in the present study may proscribe a dominant inhibitory role for CD96 relatively later time-points after antigen stimulation, while TIGIT may function earlier in T-cell activation. Upon longer term antigen-specific stimulation, we observed co-expression of CD96 not only with the inhibitor receptor TIGIT but also with the stimulatory receptor CD226 on T cells, and these coexpressing populations were enriched in proliferating $\mathrm{CD}^{+} \mathrm{T}$ cells. Given these observations, it will be critical to elucidate the relative functional inhibition upon CD155 ligation via TIGIT versus CD96 or any functional cross-talk between receptors.

Analysis of human tumors using gene expression analysis indicated that, similar to the inhibitory receptor TIGIT, elevated CD96 expression correlated with CD8 expression and 
infiltration of NK cells, although the association of T-cell markers with CD96 was stronger. The identity and functional importance of CD96 ${ }^{+}$TILs, as well as any co-expression of CD96 with TIGIT in the tumor microenvironment, remain to be determined. Previous studies of CD96 biology suggested a role in mediating NK cell adhesion and activation, and we did note a moderate correlation of CD96 expression with NK markers; however, this was not as prominent as the T cell correlation observed here.

Therapeutic targeting of the tumor immunosuppressive/co-inhibitory pathways PD-L1/PD-1 and CTLA-4 with blocking antibodies have demonstrated durable responses and improved overall survival in cancer patients. Moreover, simultaneous targeting of multiple co-inhibitory receptors (e.g. anti-PD-1 combined with anti-CTLA-4 mAbs) results in even greater antitumor immunity and anticancer effects ${ }^{1,2}$. Collectively, these data show that CD96 is predominantly expressed by human effector T cells, correlates with T-cell markers in multiple human cancers and is enriched on antigen-experienced T cells and TILs. These data support the idea that interference with CD96 signaling may be beneficial for immunotherapy against cancer and infectious disease.

\section{METHODS}

\section{Flow cytometric profiling}

PBMCs were isolated by Ficoll-Hypaque centrifugation into RPMI 1640 medium supplemented with $10 \%$ fetal calf serum (FCS) (R10 medium). Blood donors were healthy, unrelated individuals who had given written informed consent. Approval was obtained from the QIMR Berghofer Medical Research Institute Human Ethics Committee (Brisbane, Australia). All cells were stained with LIVE/DEAD fixable aqua dead cell stain. Antibody details are listed in Supplementary table 2. The white blood cell flow panel comprised CD3FITC, TCRaß-BV786, CD56-PE-Cy7, CD16-AF700, CD19-BV421, CD14-APC-Cy7, CD155-APC, CD96-PE, TIGIT-PerCP-EF710, CD226-BV711 and PD-1-BV604. The naïve/memory T-cell flow panel comprised CD4-FITC, CD8-APC-Cy7, TCRaß-BV786, CD45RA-PB, CCR7-PE-Cy7, CD96-PE, TIGIT-PerCP-EF710, DNAM-1-BV711 and PD-1BV604. The T-helper flow panel comprised CD4-FITC, TCRaß-BV786, IL17-BV421, IFNyAF700, IL4-PE-Cy7, CD96-PE, TIGIT-PerCP-EF710, DNAM-1-BV711 (or-PE) and PD-1BV6054. The Treg flow panel comprised CD3-FITC, CD4-PB, CD25-PE-Cy7, FoxP3-APC, CD127-BV786, CD96-PE, TIGIT-PerCP-EF710, DNAM-1-BV711 and PD-1-BV604. The MAIT flow panel comprised CD3-AF700, TCRYס-BV421, TCRVa7.2-FITC, CD161-PerCPCy5.5, CD96-PE, TIGIT-APC, CD226-BV711 and PD-1-BV605. All functional markers were gated relative to a fluorochrome-matched isotype control. Human cells used for mixed lymphocyte reaction assays were stained with antibodies for CD3-BV786, CD4-FITC, CD8AF700, CD14-APC-Cy7 CD96-PE, TIGIT-APC, PD-1-BV605, DNAM-BV711, both on day 0 and day 5 of the mixed lymphocyte reaction. Dendritic cells used for mixed lymphocyte reaction were stained for CD112-APC and CD155-Percp. For the tetramer experiments, $1 \mu \mathrm{g}$ of tetramer was added per test; tetramers included A1-VTEHDTLLY (A1-VTE), A2NLVPMVATV (A2-NLV), B7-RPHERNGFTVL (B7-RPH), B8-ELKRKMIYM (B8-ELK), B8RAKFKQLL (B8-RAK) and B35-HPVGEADYFEY (B35-HPV) labeled in PE or APC. Flow cytometric experiments were performed on a LSR Fortessa 5 (BD Biosciences, Franklin Lakes, NJ, USA) and analyzed using the FlowJo software (Tree Star Inc, Ashland, OR, USA). 


\section{viSNE analysis}

FCS files of PBMC samples were concatenated and viSNE analysis was performed on the resulting file utilizing Cytobank (Cytobank Inc) ${ }^{29}$. Analysis was performed on $\mathrm{CD}^{+}$cells and $\mathrm{CD}^{+} \mathrm{CD}^{+} \mathrm{T}$ cells for $\mathrm{T}$-effector subsets and $\mathrm{T}$-helper subsets, respectively. Analysis settings were iterations 1500-2000, theta 0.3 and perplexity 20-40. Generated consensus maps (viSNE maps of concatenated file) of T-cell effector subsets and T-cell helper subsets were then colored by all channels used for analysis to identify expression patterns of CD96, TIGIT, CD226 and PD-1.

\section{CyTOF profiling}

Tissue samples were obtained from patients undergoing elective surgery for colorectal cancer at Dunedin Hospital, New Zealand. The study was approved by the Health and Disability Ethics Committee (\#14/NTA/33) and all patients gave written informed consent prior to inclusion in the study in accordance with the Treaty of Helsinki. Specimens were dissected by a pathologist.

Cryopreserved tumor dissociates (TD) and PBMCs from colorectal cancer patients were used for mass cytometric analyses. Samples were maintained in sterile phosphate-buffered saline (PBS; Sigma-Aldrich, St Louis, MO, USA) at $4^{\circ} \mathrm{C}$ for no longer than 3 hours until transportation on ice. Samples were washed in sterile PBS and suspended in RPMI (Invitrogen, Carlsbad, CA, USA) with $0.5 \mathrm{mg} \mathrm{mL}^{-1}$ collagenase (Invitrogen) and incubated at $37^{\circ} \mathrm{C}, 5 \% \mathrm{CO}_{2}$, for 1 hour. The tissue was then mechanically dissociated with a sterile scalpel. The tissue suspension was removed from the well and filtered with a $70-\mu \mathrm{m}$ cell strainer (BD Falcon, Franklin Lakes, NJ) into a 50-mL Falcon tube. Live immune cells were enriched using a three-layer Ficoll-Paque Plus (GE Healthcare, Chicago, IL, USA) gradient. Five milliliters of $75 \%$ Ficoll, 25\% RPMI-10 [RPMI supplemented with $100 \mu \mathrm{g} \mathrm{mL}^{-1}$ penicillin, $100 \mu \mathrm{g} \mathrm{mL}^{-1}$ streptomycin and $55 \mu \mathrm{m}$ 2-mercaptoethanol (all from Invitrogen)] was layered on top of $5 \mathrm{~mL} 100 \%$ Ficoll. A quantity of $2.5 \mathrm{~mL}$ cell suspension in RPMI-10 was layered above this. Ficoll gradients were then centrifuged at $800 \mathrm{~g}$ for $20 \mathrm{~min}$ with no brake. PBMCs were also isolated using Ficoll separation. The buffy coat layer containing live immune cells was then carefully removed using a Pasteur pipette. Cells were frozen in freezing media (90\% FCS (PAA Laboratories), 10\% DMSO (Sigma-Aldrich) in liquid nitrogen for storage. Samples were transported to Sydney on dry ice for a maximum of $12 \mathrm{~h}$ and stored at $-80^{\circ} \mathrm{C}$ prior to staining.

Samples were rapidly thawed, washed in FACS media (1× DPBS supplemented $1 \%$ FCS and $0.05 \%$ EDTA) and counted using Trypan Blue exclusion viability dye. Metal-conjugated antibodies used in analysis are presented in the Key Resource Table. For some markers, fluorophore-conjugated antibodies were used as primary antibodies, followed by secondary labeling with anti-fluorophore metal-conjugated antibodies. Antibodies were either purchased from Fluidigm or conjugated in-house using MaxPar X8 reagent kits (Fluidigm), according to the manufacturer's protocol (Supplemental table 2). Conjugated antibodies were titrated for optimal concentration prior to use. Surface and intracellular antibody staining cocktail master mixes were prepared prior to each experiment. All antibody preparation and quality control were carried out by the Ramaciotti Facility for Human Systems Biology, Sydney. To most accurately compare protein expression levels between PBMC and TD we utilized a CD45-based barcoding strategy ${ }^{30}$, whereby a given patient's TD and PBMCs were first stained separately with CD45-Pd104 and CD45-Pd110, separately then washed and combined for subsequent antibody staining steps. Cells were stained for 
mass cytometry analyses as described ${ }^{31}$. Briefly, cells were stained with $1.25 \mu \mathrm{m}$ Cell-IDTM Cisplatin in PBS (Fluidigm) 3 min at room temperature and quenched by rapid addition of FCS. Cells were then washed twice in FACS buffer and then stained with a fluorophoreconjugated antibody cocktail for $20 \mathrm{~min}$ at $4^{\circ} \mathrm{C}$. Following wash with FACS buffer, cells were stained with a metal-conjugated surface stain antibody cocktail for 20 min at $4^{\circ} \mathrm{C}$. Cells were then fixed and permeabilized using the FoxP3 Transcription Factor Staining Buffer Set, according to the manufacturer's protocol (eBiosciences, Santa Clara, CA, USA). Cells were subsequently stained with a metal-conjugated intracellular antibody cocktail for 40 min at $4^{\circ} \mathrm{C}$. Cells were then washed twice, once in Perm/Wash buffer and once in FACS buffer. Cells were then fixed overnight in $4 \%$ paraformaldehyde solution containing DNA Intercalator (0.125 $\mu \mathrm{M}$ iridium-191/193; Fluidigm). Prior to acquisition, cells were washed once in FACS and twice in $\mathrm{dH} 2 \mathrm{O}$. Cells were then diluted to $8 \times 10^{5}$ cells $\mathrm{mL}^{-1}$ in $\mathrm{dH}_{2} \mathrm{O}$ containing $10 \% \mathrm{EQ}$ Four Element Calibration Beads (Fluidigm) and filtered. Cells were acquired at a rate of 200-400 cells s ${ }^{-1}$ using a CYTOF 2 Helios upgraded mass cytometer (Fluidigm). Flow Cytometry Standard (FCS) files were normalized to EQ bead signal and were then analyzed using FlowJo v10.2 (Tree Star Inc). TD and PBMC leukocytes were debarcoded manually in FlowJo.

\section{NanoString profiling}

CD96 ${ }^{\text {high }}$ and CD96 low cells were sorted from memory $\mathrm{CD}^{+}$and $\mathrm{CD} 8^{+} \mathrm{T}$ cells using an Aria III (BD Biosciences) with FACSDiva (BD Biosciences). Total RNA from each T-cell population was extracted using RNAzol RT (Astral Scientific), isopropanol (Sigma Aldrich) and ethanol (Sigma Aldrich). Briefly, cell lysate was mixed with UltraPure $\mathrm{H}_{2} \mathrm{O}$ (Sigma Aldrich) and centrifuged to separate RNA from other cell components. The clear liquid phase is then diluted with isopropanol and centrifuged to pellet RNA, washed with $75 \%$ ethanol, dried and resuspended in $\mathrm{H}_{2} \mathrm{O}$ for downstream use. Overnight nCounter codeset hybridization was performed as per the manufacturer's instruction using $100 \mathrm{ng}$ of RNA, quantified by a Nanodrop 1000 (Thermo Scientific). The 135-plex custom nCounter codeset covered key genes involved in human T-cell recognition, survival, migration, adhesion, cytokine/chemokine secretion, differentiation and exhaustion. Following hybridization, samples were purified using an nCounter Prep Station before quantification with the nCounter Digital Analyzer. Multivariate analysis and network analysis were conducted using GraphPad PRISM (GraphPad software) and GMine software ${ }^{12 .}$

\section{T-cell kinetics profiling}

$\mathrm{CD}^{+} \mathrm{T}$ cells were purified from the PBMC of genetically unrelated donors using a negative selection Pan T cell Isolation Kit (Miltenyi Biotec). Isolated CD3 ${ }^{+} \mathrm{T}$ cells were activated using anti-CD3/CD28 Dynabeads (ThermoFisher Scientific) at the final ratio of 1 bead: 5 cells in R10 medium. 200000 activated T cells were plated in a V-bottom 96-well microtiter plate and harvested after 3 h, 6 h, day 1 , day 3 , day 5 , day 7 and day 10 after stimulation. Media was replaced at day 5 and cells split into two wells. Nonstimulated T cells were used as controls. Prior to flow cytometric analysis and RNA extraction, Dynabeads beads were removed via magnetic separation. T cells were stained with anti-CD3-BV786, CD4-PB, CD8AF700, CD96-PE, TIGIT-APC, CD226-BV711 and PD1 BV605. Relevant isotype controls were used in parallel. Dead cells were excluded by LIVE/DEAD fixable aqua dead cell stain. Flow cytometry was performed on a LSR Fortessa 4 (BD Biosciences). 


\section{Real-time PCR}

RNA was isolated from flow cytometry-based sorted human T cells by an RNAeasy microkit (Qiagen) as per the manufacturer's instructions. One hundred nanograms of mRNA was used to synthesize cDNA using a sensiFAST cDNA synthesis Kit (Bioline). Predesigned Prime Time TaqMan qPCR assays were used to amplify mRNA and measure gene expression for CD96 (isoform 1 and 2), TIGIT, PD-1 and CD226 (IDT Technologies) using 6 -carboxyfluorescein as a reporter on the ABI ViiA 7 (Applied Biosystems) Real-Time PCR system. CDNA was denatured and amplified at $95^{\circ} \mathrm{C}$ for $5 \mathrm{~s}$ and then $60^{\circ} \mathrm{C}$ for $30 \mathrm{~s}$ for 42 cycles. Gene expression levels were normalized to the housekeeping gene $\beta-2$ microglobulin (2(Ct value $\beta 2 \mathrm{~m}-\mathrm{Ct}$ value gene of interest)).

\section{Mixed lymphocyte reaction}

$\mathrm{CD}^{+} 4^{+}$monocytes were isolated by positive selection using CD14 microbeads (Miltenyi Biotec) and cultured in vitro for 7 days with $300 \mathrm{IU} \mathrm{mL} \mathrm{mL}^{-1} \mathrm{IL}-4$ and $1000 \mathrm{IU} \mathrm{mL} \mathrm{mL}^{-1} \mathrm{GM}-\mathrm{CSF}$ (Preprotech Inc) to generate dendritic cells. Untouched T cells were isolated from PBMC with Rosette Sep (StemCell Technologies). Briefly, blood was collected and Rosette Sep ${ }^{\mathrm{TM}}$ cocktail added and incubated for $20 \mathrm{~min}$ at room temperature. After dilution of the sample, a density gradient was performed and purified $T$ cells collected from the enriched cell layer. CD3 ${ }^{+} \mathrm{T}$ cells $\left(1 \times 10^{5}\right)$ and allogeneic dendritic cell $\left(1 \times 10^{4}\right)$ were cocultured with in R10 medium. Flow cytomety was performed on a BD LSR Fortessa 4 (BD Biosciences) instrument and analyzed using the FlowJo software (Tree Star Inc).

\section{T-cell priming using LCL feeders}

Short-term Epstein-Barr virus-specific T-cells cultures were established using LCL as stimulator cells. Donors were healthy adults and Epstein-Barr virus seropositive. Briefly, $2.5 \times 10^{5}$ purified $\left(\mathrm{CD}^{+}\right.$or $\left.\mathrm{CD}^{+}\right)$T cells were seeded with irradiated autologous LCLs in a responder to stimulator ratio of 30:1 in a U-bottom 96-well microtiter plate in R10 medium supplemented with $45 \mathrm{IU} \mathrm{mL} \mathrm{m}^{-1}$ recombinant human IL-2 (Proleukin; Chiron) at day 4. CD3 ${ }^{+}$ $T$ cells were purified by negative selection using RosetteSet $T$ cell enrichment cocktail (STEMCELL Technologies). T cells were stained with CellTrace Violet (Thermo Fisher) in order to check proliferating $\left(\mathrm{CTV}^{-}\right)$versus nonproliferating $\left(\mathrm{CTV}^{+}\right)$populations after 7 days of stimulation with autologous LCL cells. Flow cytometric measures were performed on a BD LSR Fortessa 4 (BD Biosciences) instrument and analyzed using the FlowJo software (Tree Star Inc).

\section{Bioinformatics}

RNAseq data from the TCGA breast cancer and endometrial carcinoma project were obtained from the UCSC Cancer Genomics Hub. Raw RNA-seq reads were trimmed for adapter sequences using Cutadapt and aligned to the GRCH37 assemble using STAR aligner. Quality control metrics were computed using RNA-SeQC and expression was estimated using RSEM. The samples were normalized using library size estimation and corrected for differences in RNA composition using the method trimmed mean of M-values implemented in the edgeR package ${ }^{32}$. To determine the correlation between expression of the CD96 and TIGIT genes with other genes of interest, we utilized RNA-sequencing and data were normalized using the edgeR package. Spearman's rank correlation was estimated on the normalized counts. We considered $\rho>0.75$ to be indicator of strong correlation, $\rho<0.75$ but $\rho>0.5$ for moderate correlation and $\rho<0.5$ to be indicator of weak correlation. 
For calculation of CD96/CD3E ratios, we first transformed our data on to log2 scale and then estimated the log2 ratio of the normalized counts for CD96 and CD3E. A similar method was performed for other gene pairs. To calculate the differences in log ratios between the normal and tumor samples, we applied a linear regression model using standard $\mathrm{R}$ function. A $P$ value of $<0.01$ was considered as evidence of significance between normal and tumor samples.

\section{ACKNOWLEDGMENTS}

The authors acknowledge the specimen donors and research groups associated with SKCM samples acquisition and analysis associated with the Cancer Genome Atlas Research Network (TCGA). The project was funded by a National Health and Medical Research Council of Australia (NH\&MRC) Development Grant (1093566). MJS is supported by a Senior Principal Research Fellowship (1078671) and JJM by an NH\&MRC Career Development Fellowship (1131732). RAK is the recipient of the New Zealand Society for Oncology-Roche Translational Cancer Research Fellowship (2016).

\section{CONFLICT OF INTERESTS}

MJS, WCD and JJM have been supported by a scientific research agreement with BristolMyers Squibb. MJS was a consultant for Kymab, F-star and Arcus Biosciences. WCD has received a consulting honorarium from Amgen. 


\section{REFERENCES}

1. Larkin, J, Chiarion-Sileni, V, Gonzalez, R, et al. Combined nivolumab and ipilimumab or monotherapy in untreated melanoma. N Engl J Med 2015; 373: 23-34.

2. Hodi, FS, Chesney, J, Pavlick, AC, et al. Combined nivolumab and ipilimumab versus ipilimumab alone in patients with advanced melanoma: 2-year overall survival outcomes in a multicentre, randomised, controlled, phase 2 trial. Lancet Oncol 2016; 17: 1558- 1568.

3. Blake, SJ, Dougall, WC, Miles, JJ, Teng, MW, Smyth, MJ. Molecular pathways: targeting CD96 and TIGIT for Cancer Immunotherapy. Clin Cancer Res 2016; 22: 5183- 5188.

4. Gilfillan, S, Chan, CJ, Cella, M, et al. DNAM-1 promotes activation of cytotoxic lymphocytes by nonprofessional antigen-presenting cells and tumors. J Exp Med 2008; 205: 2965- 2973.

5. Iguchi-Manaka, A, Kai, H, Yamashita, Y, et al. Accelerated tumor growth in mice deficient in DNAM-1 receptor. J Exp Med 2008; 205: 2959- 2964.

6. Kurtulus, S, Sakuishi, K, Ngiow, SF, et al. TIGIT predominantly regulates the immune response via regulatory T cells. J Clin Invest 2015; 125: 4053- 4062.

7. Johnston, RJ, Comps-Agrar, L, Hackney, J, et al. The immunoreceptor TIGIT regulates antitumor and antiviral CD8(+) T cell effector function. Cancer Cell 2014; 26: 923- 937.

8. Chan, CJ, Martinet, L, Gilfillan, S, et al. The receptors CD96 and CD226 oppose each other in the regulation of natural killer cell functions. Nat Immunol 2014; 15: 431-438.

9. Zhang, Q, Bi, J, Zheng, X, et al. Blockade of the checkpoint receptor TIGIT prevents NK cell exhaustion and elicits potent anti-tumor immunity. Nat Immunol 2018; PMID: 29915296 [E-pub ahead of print].

10. Chauvin, JM, Pagliano, O, Fourcade, J, et al. TIGIT and PD-1 impair tumor antigenspecific CD8(+) T cells in melanoma patients. J Clin Invest 2015; 125: 2046-2058.

11. Blake, S J, Stannard, K, Liu, J, et al. Suppression of metastases using a new lymphocyte checkpoint target for cancer immunotherapy. Cancer Discov 2016; 6: 446- 459.

12. Proietti, C, Zakrzewski, M, Watkins, TS, et al. Mining, visualizing and comparing multidimensional biomolecular data using the Genomics Data Miner (GMine) WebServer. Sci Rep 2016; 6: 38178.

13. Weber, JS, D'Angelo, SP, Minor, D, et al. Nivolumab versus chemotherapy in patients with advanced melanoma who progressed after anti-CTLA-4 treatment (CheckMate 037): a randomised, controlled, open-label, phase 3 trial. Lancet Oncol 2015; 16: 375- 384.

14. Hodi, FS. Improved survival with ipilimumab in patients with metastatic melanoma. $N$ Engl J Med 2010; 363: 711- 723.

15. Postow, MA, Chesney, J, Pavlick, AC, et al. Nivolumab and ipilimumab versus ipilimumab in untreated melanoma. N Engl J Med 2015; 372: 2006- 2017.

16. Das, M, Zhu, C, Kuchroo, VK. Tim-3 and its role in regulating anti-tumor immunity. Immunol Rev 2017; 276: 97- 111.

17. Vilgelm, AE, Johnson, DB, Richmond, A. Combinatorial approach to cancer immunotherapy: strength in numbers. J Leukoc Biol 2016; 100: 275- 290.

18. Nowak, EC, Lines, JL, Varn, FS, et al. Immunoregulatory functions of VISTA. Immunol Rev 2017; 276: 66- 79.

19. Podojil, JR, Miller, SD. Potential targeting of B7-H4 for the treatment of cancer. Immunol Rev 2017; 276: 40-51. 
20. Dougall, WC, Kurtulus, S, Smyth, MJ, Anderson, AC. TIGIT and CD96: new checkpoint receptor targets for cancer immunotherapy. Immunol Rev 2017; 276: $112-120$.

21.Brooks, J, Fleischmann-Mundt, B, Woller, N, et al. Perioperative, spatiotemporally coordinated activation of $T$ and NK cells prevents recurrence of pancreatic cancer. Cancer Res 2018; 78: 475- 488.

22. Topalian, SL, Drake, CG, Pardoll, DM. Immune checkpoint blockade: a common denominator approach to cancer therapy. Cancer Cell 2015; 27: 450- 461.

23. Bevelacqua, V, Bevelacqua, Y, Candido, S, et al. Nectin like-5 overexpression correlates with the malignant phenotype in cutaneous melanoma. Oncotarget 2012; 3: 882- 892.

24. Nakai, R, Maniwa, Y, Tanaka, Y, et al. Overexpression of Necl-5 correlates with unfavorable prognosis in patients with lung adenocarcinoma. Cancer Sci 2010; 101: $1326-1330$.

25. Masson, D, Jarry, A, Baury, B, et al. Overexpression of the CD155 gene in human colorectal carcinoma. Gut 2001; 49: 236- 240.

26. Soriani, A, Zingoni, A, Cerboni, C, et al. ATM-ATR-dependent up-regulation of DNAM-1 and NKG2D ligands on multiple myeloma cells by therapeutic agents results in enhanced NK-cell susceptibility and is associated with a senescent phenotype. Blood 2009; 113: 3503- 3511.

27. Li, X-Y, Das, I, Lepletier, A, et al. CD155 loss enhances tumor suppression via combined host and tumor-intrinsic mechanisms. J Clin Invest 2018; 128: 2613-2625.

28. Guillerey, C, Nakamura, K, Vuckovic, S, Hill, GR, Smyth, MJ. Immune responses in multiple myeloma: role of the natural immune surveillance and potential of immunotherapies. Cell Mol Life Sci 2016; 73: 1569- 1589.

29. Kotecha, N, Krutzik, PO, Irish, JM. Web-based analysis and publication of flow cytometry experiments. Curr Protoc Cytom 2010;Chapter 10: Unit10 17.

30. Mei, HE, Leipold, MD, Schulz, AR, Chester, C, Maecker, HT. Barcoding of live human peripheral blood mononuclear cells for multiplexed mass cytometry. J Immunol 2015; 194: 2022- 2031.

31. McGuire, HM, Shklovskaya, E, Edwards, J, et al. Anti-PD-1-induced high-grade hepatitis associated with corticosteroid-resistant T cells: a case report. Cancer Immunol Immunother 2018; 67: 563- 573.

32. Robinson, MD, McCarthy, DJ, Smyth, GK. edgeR: a bioconductor package for differential expression analysis of digital gene expression data. Bioinformatics 2010; 26: $139-140$. 


\section{FIGURE 1}

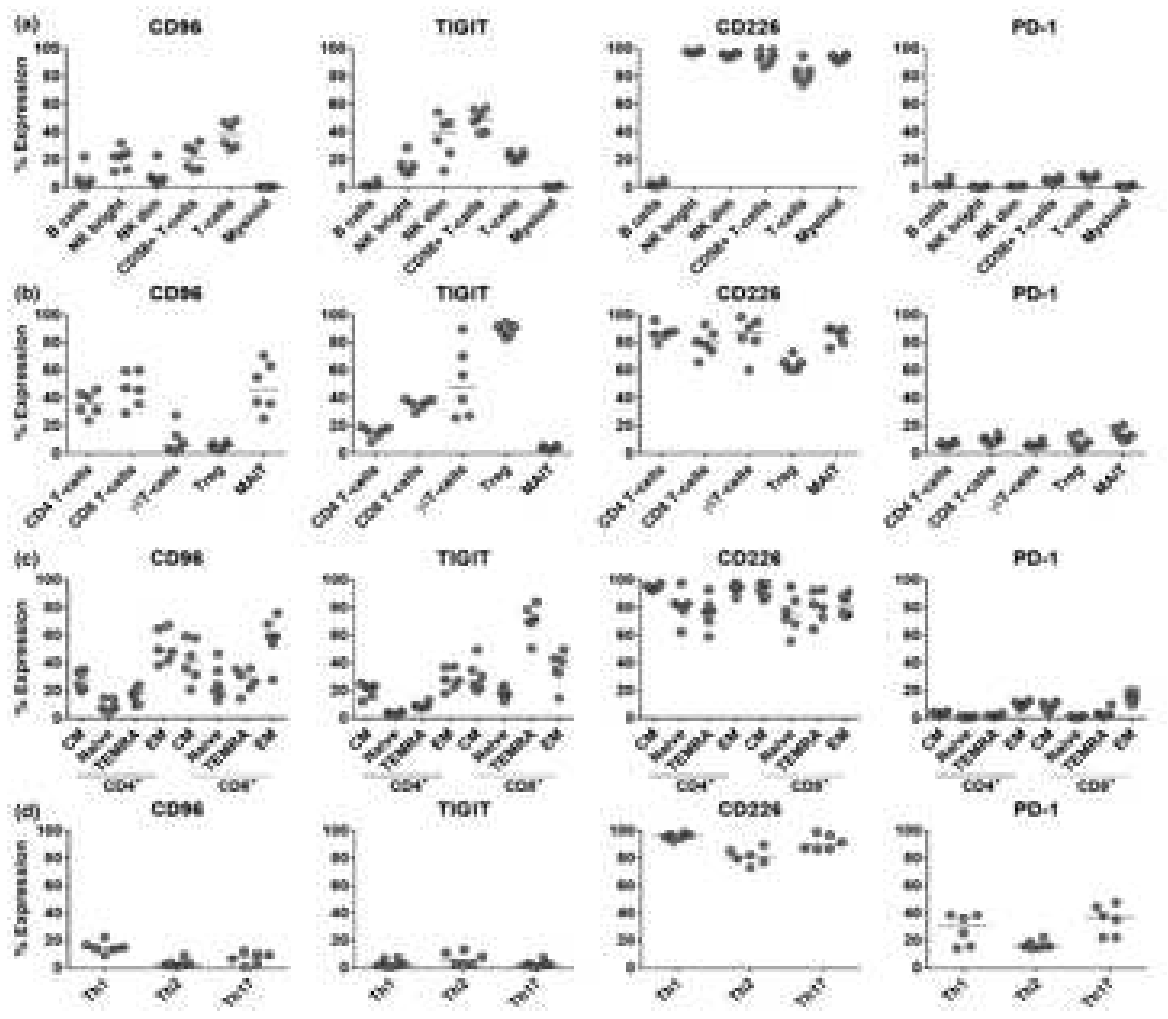

Human CD96 expression is compartmentalized by lymphocyte lineage. PBMC from six healthy individuals were examined for CD96, TIGIT, CD226 and PD-1 expression. (a) White blood cell panel gating B cells, NKbright cells, NKdim cells, CD56+ T cells, T cells and myeloid cells. (b) T-cell subtype panels gated on CD4+ T cells, CD8+ T cells, $ү \delta ~ T$ cells, Treg and MAIT cells. (c) T-cell effector panel gated on naïve, central memory (CM), EM and TEMRA for CD4+ and CD8+ lineages. (d) Th subtype panel gated on Th1, Th2 and Th17 cells post-mitogen stimulation. Each immune checkpoint molecule was quantified relative to a fluorophore-matched isotype control. Percentage positive values are shown. The experiment was conducted once. Data were acquired on a BD LSR Fortessa-5 and analyzed using the FlowJo software. Each data point represents the percentage of cells positive from and individual PBMC donor. 


\section{FIGURE 2}
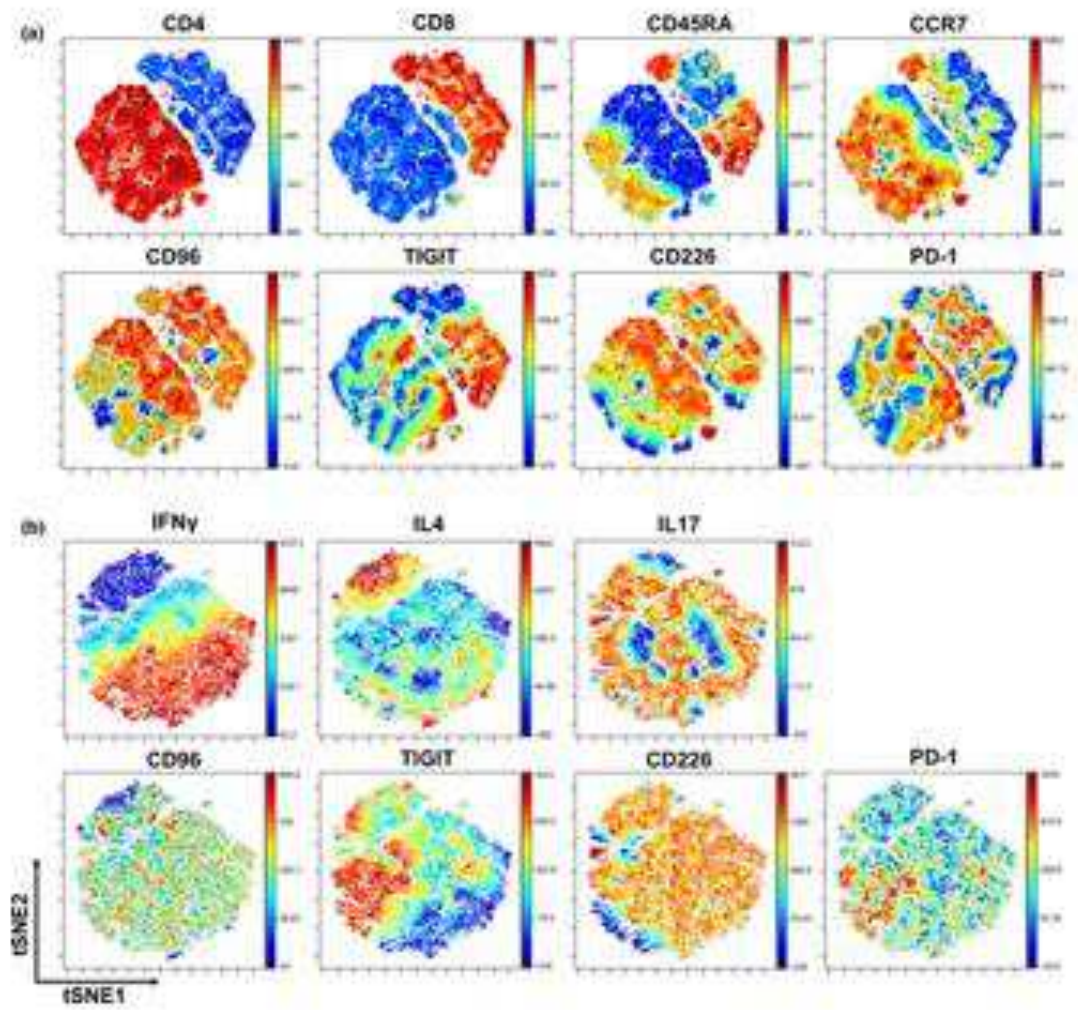

CD96 is co-expressed with CD226. (a) viSNE plots showing CD4, CD8, CD45RA, CCR7, CD96, TIGIT, CD226 and PD-1 co-expression from six concatenated PBMC samples gated on CD3+ T cells. The experiment was conducted once. (b) viSNE plots showing IFNy, IL4, IL17, CD96, TIGIT, CD226 and PD-1 co-expression from six concatenated PBMC samples gated on $\mathrm{CD} 3+/ \mathrm{CD} 4+\mathrm{T}$ cells that are cytokine positive. IFNy, IL4 and IL17 plots were gated on phorbol myristate acetate/ionomycin-activated CD4+ T cells and cytokine-negative cells have been removed from the analysis. Each phenotypic marker was quantified relative to a fluorophore-matched isotype control. FCS files were analyzed using Cytobank. t-distributed stochastic neighbor embedding (tSNE)-1 and tSNE-2 were used for embedding highdimensional objects in two dimensions in such a way that similar objects are modeled by nearby points and dissimilar objects are modeled by distant points. 


\section{FIGURE 3}
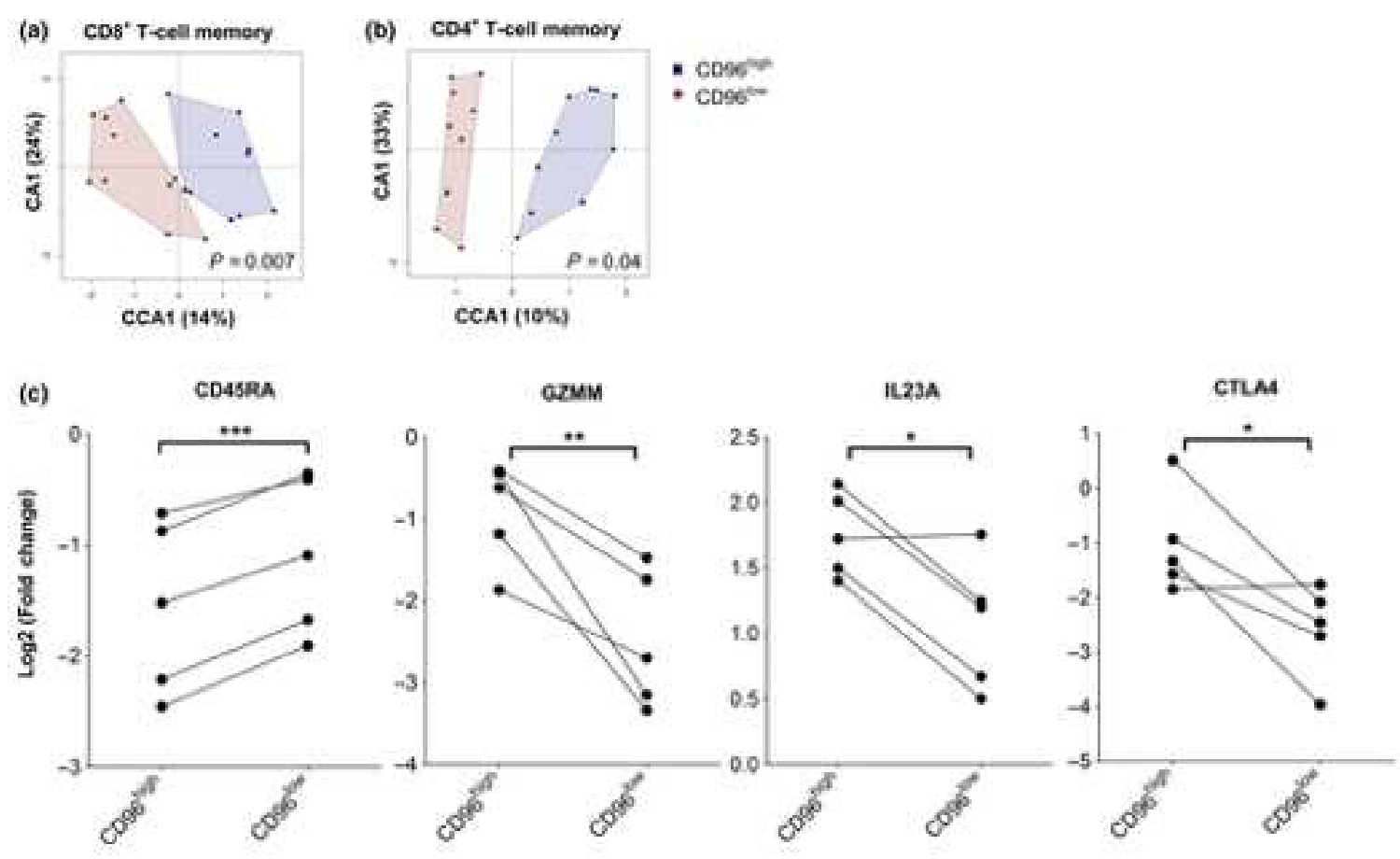

T-cells expressing CD96 exhibit a distinct transcriptional landscape. CD96high and CD96low cells were sorted from effector memory (CD45RA-/CCR7-) CD4+ and CD8+ T cells to high purity from 10 individuals. RNA was extracted and run on a 135-plex nCounter codeset. NanoString gene expression was normalized and genes filtered to exclude those with an average expression value <mean +2 SD of the negative controls. Significant, differentially expressed genes between populations are shown (Supplemental tables $2 \& 3$ ). CCA for CD8+ T cells (a) and CD4+ T cells (b) were determined using the Gmine software.12 (c) CD96high and CD96low cells were sorted from effector memory (CD45RA-/CCR7-) CD8+ T cells from five donors and stimulated with phorbol myristate acetate/ionomycin for $4 \mathrm{~h}$. To determine differences in functional output, we used log2 of the fold change following activation between CD96high and CD96low CD8+ $T$ cells. Four statistically significant examples are shown $\left({ }^{*} \mathrm{P}<0.05\right.$; ${ }^{* *} \mathrm{P}<0.01$; $\left.{ }^{* * *} \mathrm{P}<0.001\right)$. A support vector machine (SVM) trained on the transcriptional profiles of either CD96high or CD96low T cells and evaluated by leave-one-out cross-validation achieved $95 \%$ and $80 \%$ accuracy in predicting CD96 status for CD8+ and CD4+ T cells, respectively. 


\section{FIGURE 4}
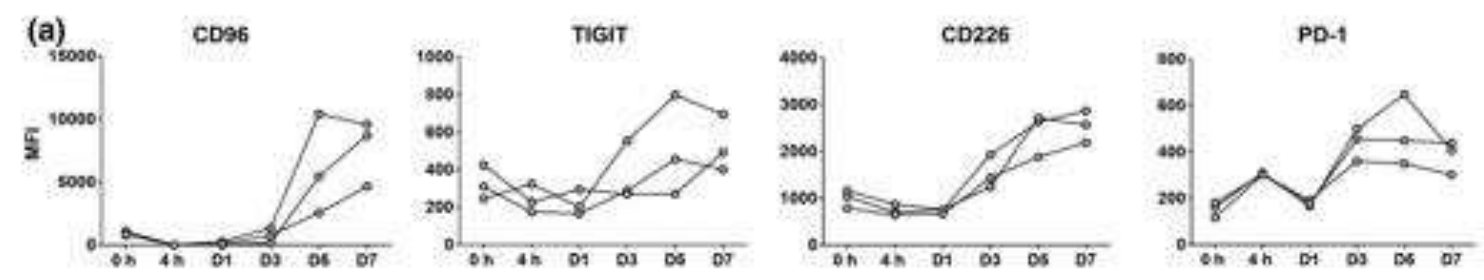

(b)

CD96

TiGr

C0226

PD-1

C069
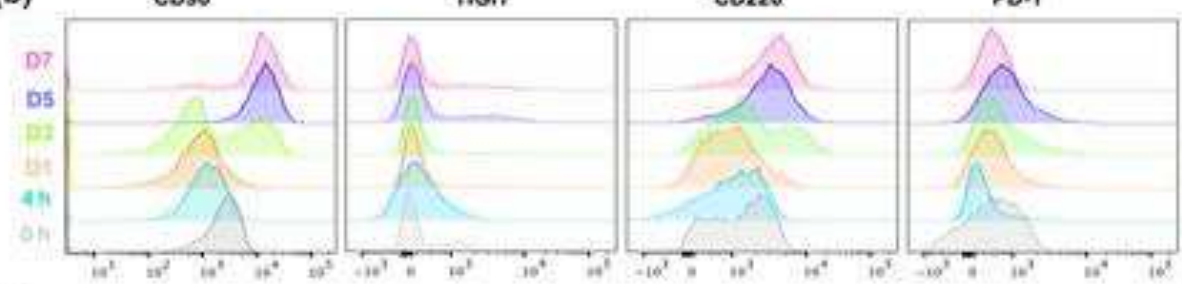

(c)

CD96

TIGT
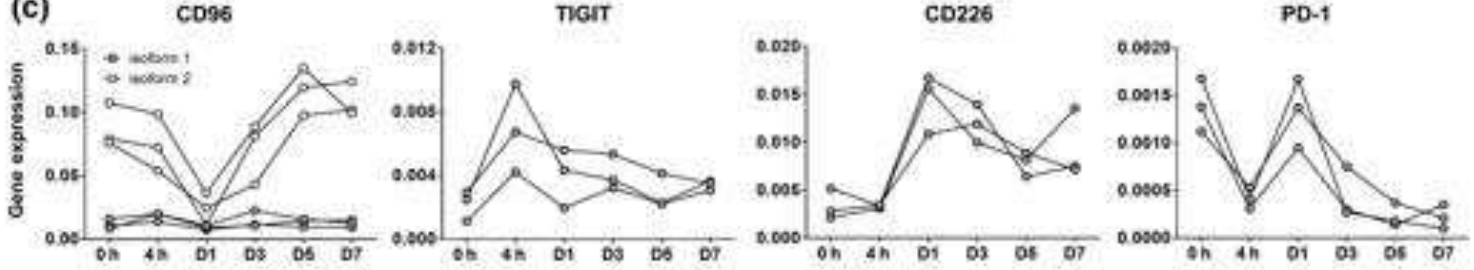

CD96 exhibits dynamic T-cell activation kinetics. CD3+ $T$ cells isolated from PBMC from three donors were stimulated with CD3/CD28 microbeads and CD96, TIGIT, CD226 and PD-1 surface expression measured by flow cytometry at $4 \mathrm{~h}$, day $1,3,5,7$ and 10 postactivation. Receptor expression (plotted as percentage positive values) are shown (a) and representative histograms are shown in b. (c) The same in vitro cultures were also profiled for mRNA expression of CD96 (isoform 1 and 2), TIGIT, CD226 and PD-1. Data are presented as relative gene expression to $\beta-2$ microglobulin. 


\section{FIGURE 5}
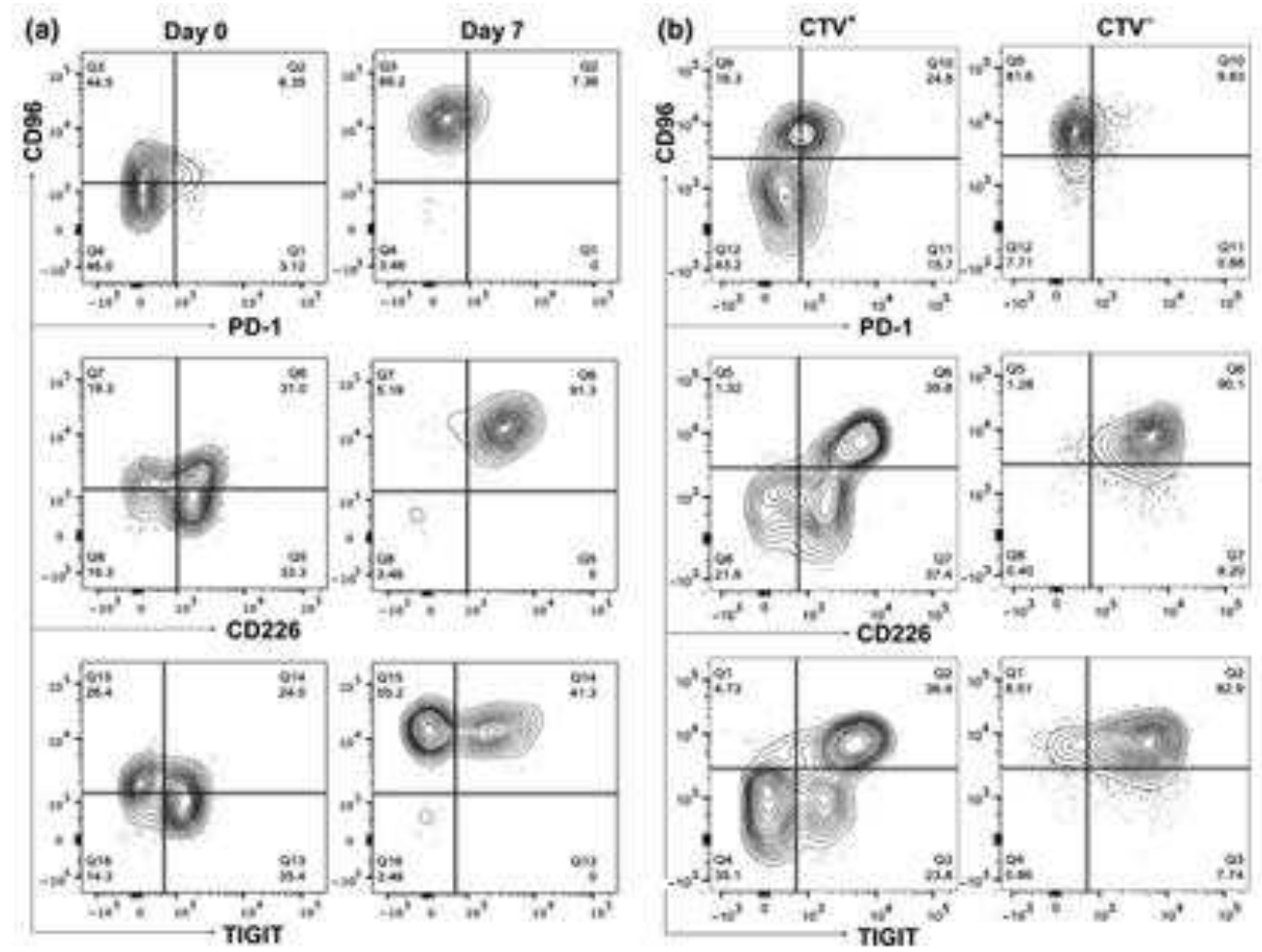

Co-expression of CD96 and PD-1, TIGIT and CD226/DNAM-1 after T-cell activation. The expression of CD96, CD226, TIGIT and PD-1 was assessed on CD8+ T cells after activation using different stimuli. (a) Representative flow cytometry dot plots of CD8+ T cells measured ex vivo and after 7 days of activation with CD3/CD28 beads. (b) Representative flow cytometry dot plot of CTV+ and CTV- CD8+ T cells activated on coculture with autologous LCL cells for 7 days. Percentage positive values are shown. Data were acquired on a BD LSR Fortessa-5 and analyzed using FlowJo software. 


\section{FIGURE 6}
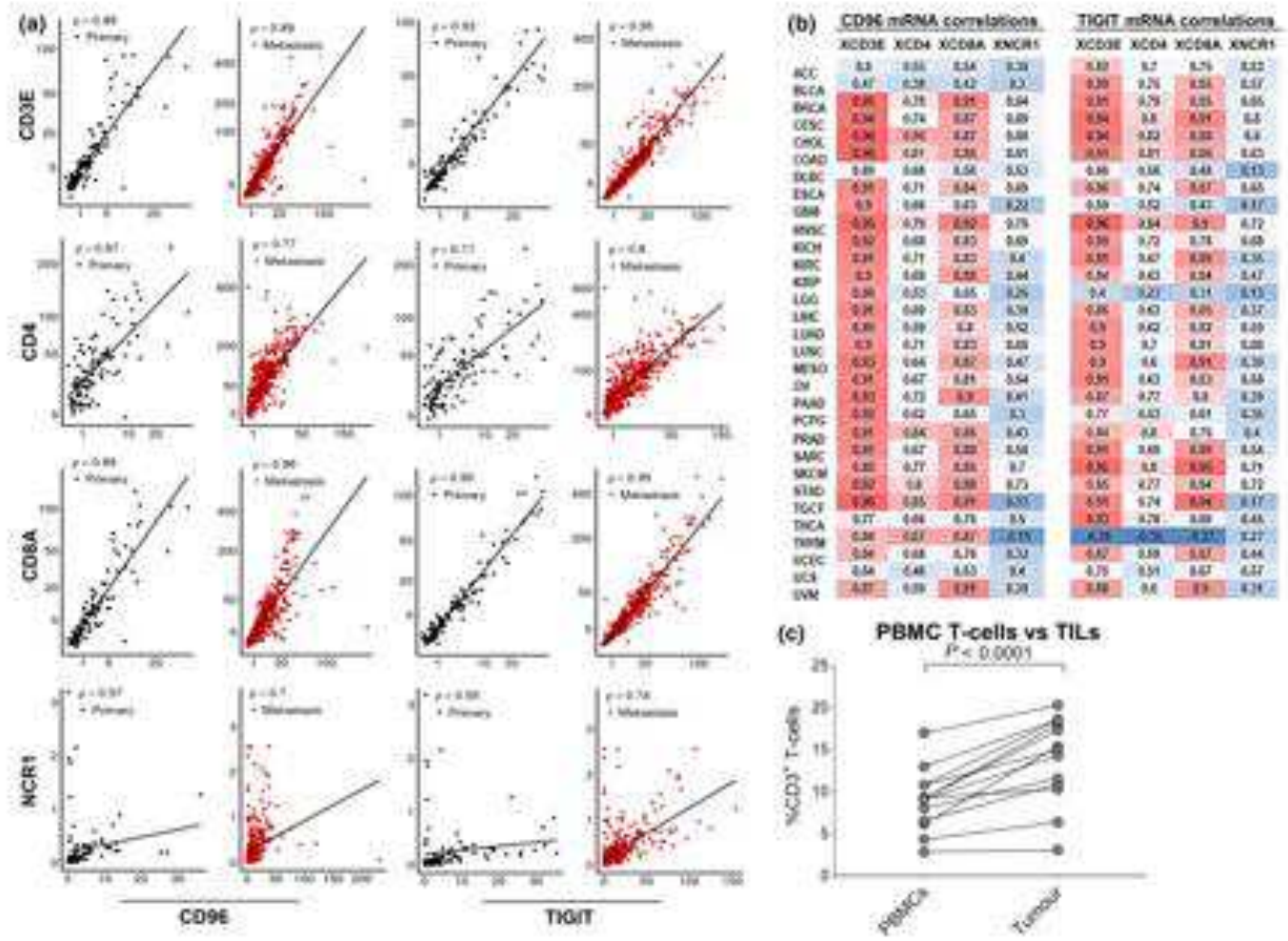

CD96 expression is strongly correlated with T-cell markers in human cancer. (a) Correlation of CD96 or TIGIT with CD3E, CD4, CD8A or NCR1 in skin cutaneous melanoma (SKCM). Scatter plots show per-gene count data normalized by library size. A $\rho>0.75$ to be indicator of strong correlation and $\rho<0.75$ but rho $>0.5$ is an indicator of moderate correlation and $\rho<0.5$ to be indicator of weak correlation. Analysis included primary tumors in black $(n=103)$ and metastatic tumors in red $(n=368)$. (b) CD96 and TIGIT gene expression is strongly correlated with T-cell markers in TCGA datasets of human cancer. Gene expression analyses of human cancers were performed as described (in Methods) for all available and Spearman's correlations for CD96 or TIGIT with T cell (CD3E, CD4, CD8) and NK cell (NCR1) markers are shown. Analysis of each of the 31 tumor types available from TCGA indicate that the correlation of CD96 and TIGIT with T-cell markers is consistently observed, with the correlation with CD8A being particularly strong (24/31 tumor types demonstrating a Spearman's correlation coefficient $>0.75$ for CD96 and 20/31 tumor types demonstrating a Spearman's correlation coefficient $>0.75$ for TIGIT). (c) CD96 expression on CD3+ T cells in paired PBMC and 13 colorectal cancer patients. 


\section{SUPPLEMENTARY FIGURE 1}

a

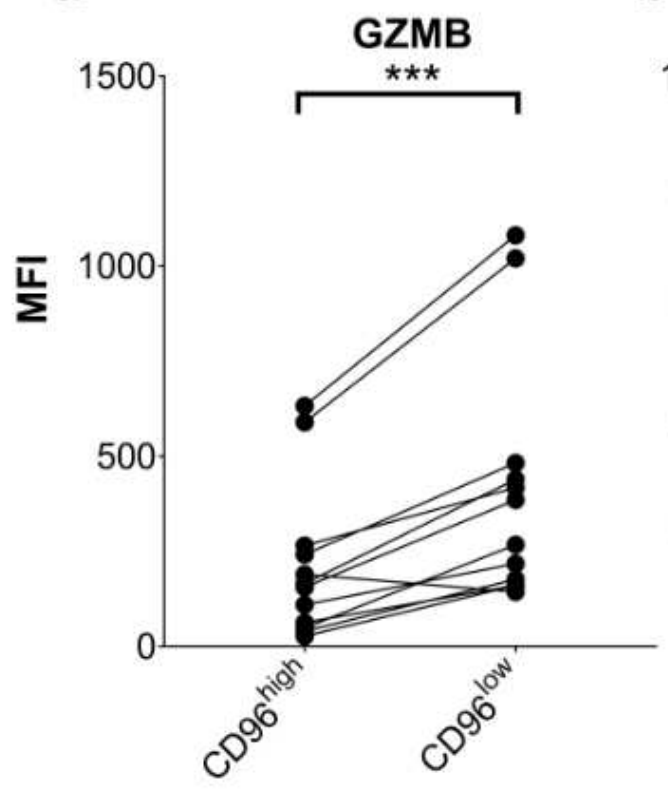

b

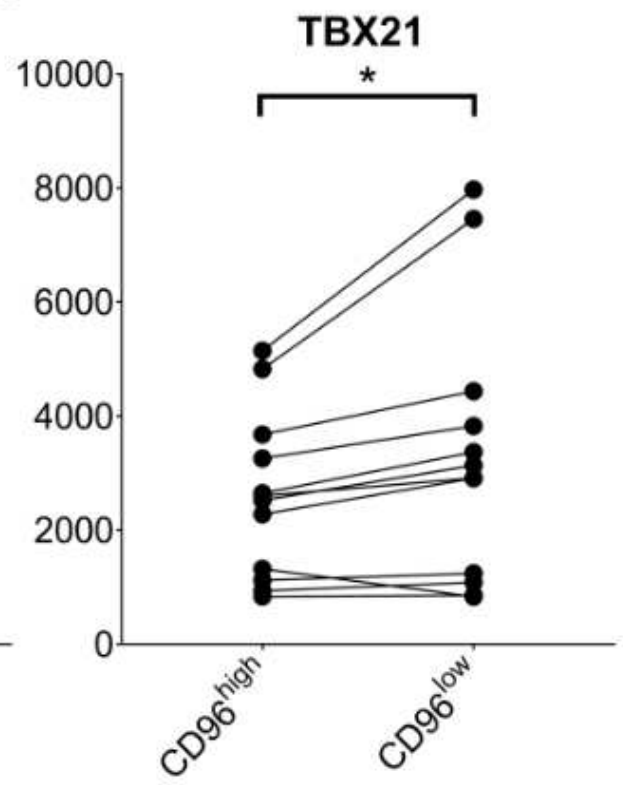

\section{SUPPLEMENTARY FIGURE 2}

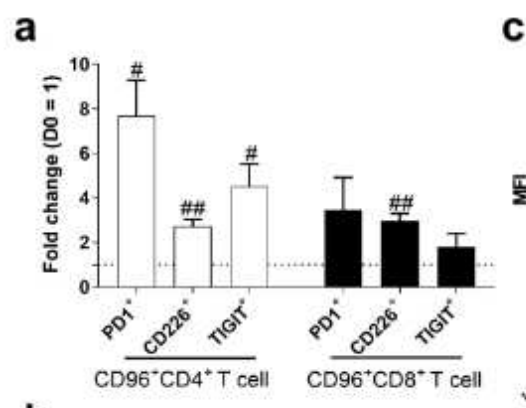

C

b
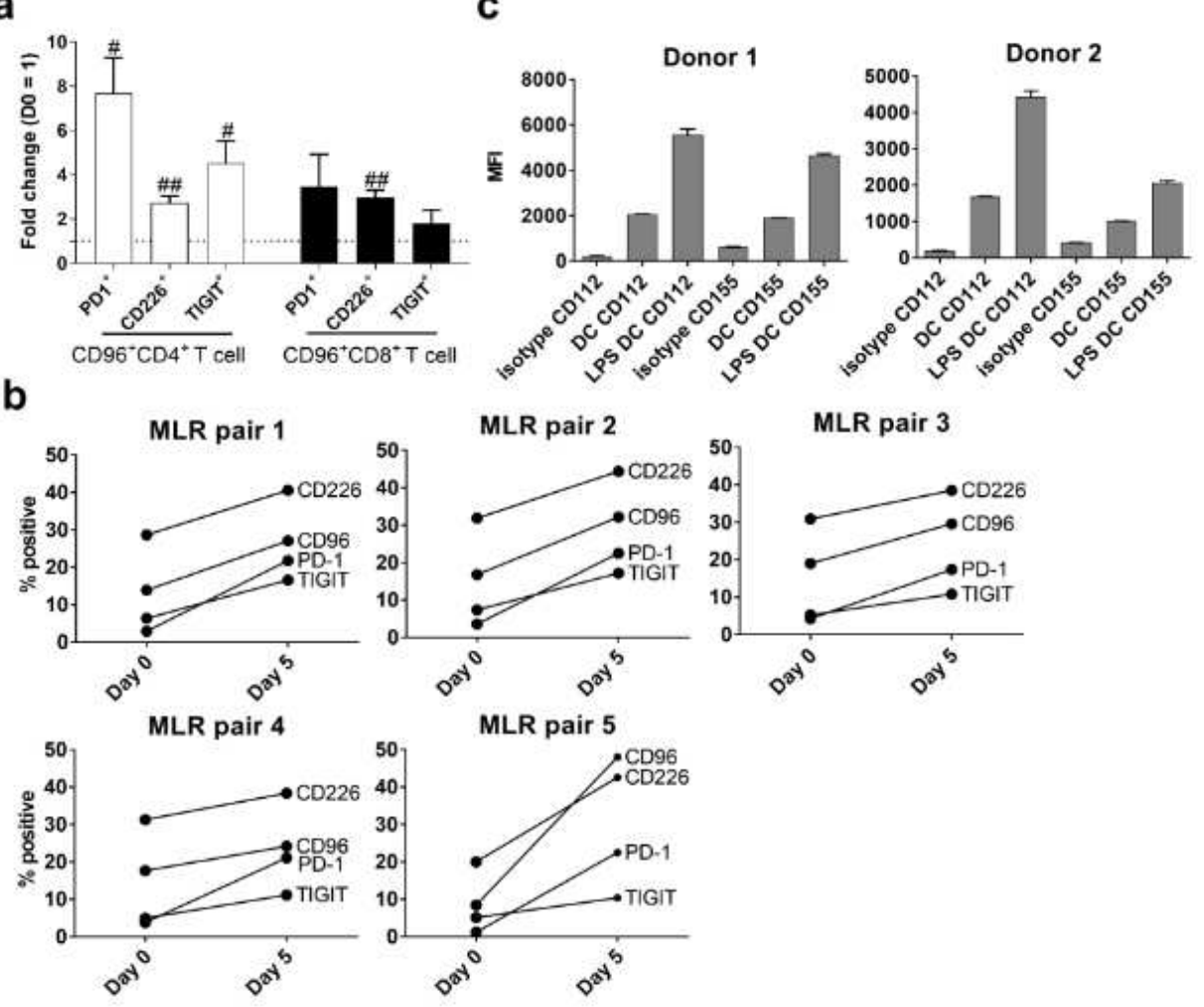


\section{SUPPLEMENTARY FIGURE 3}

a
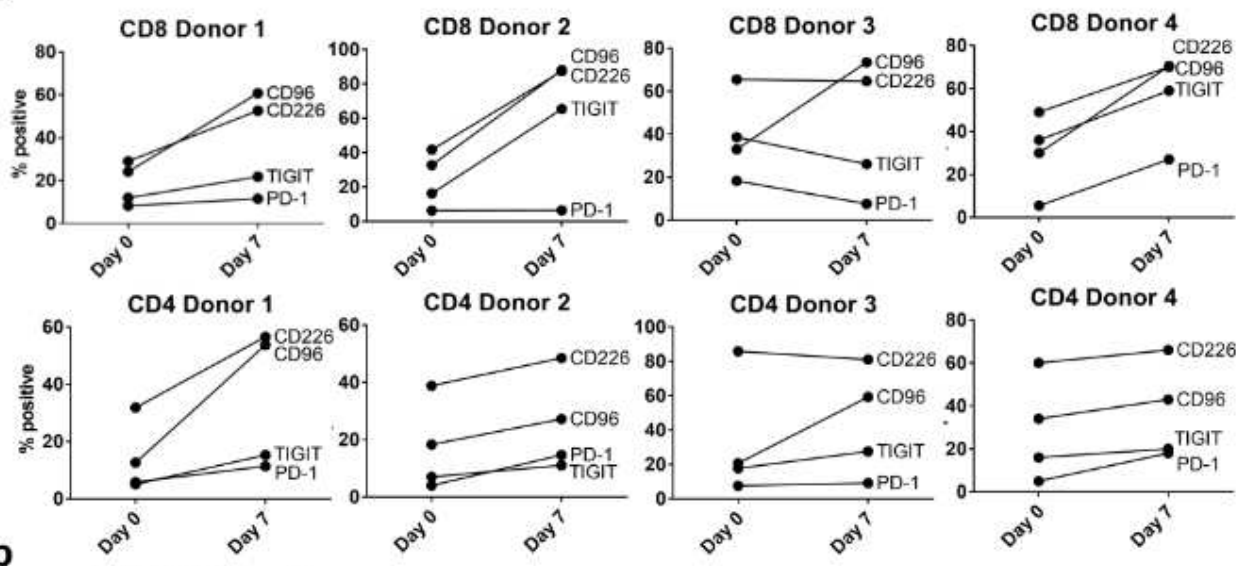

b

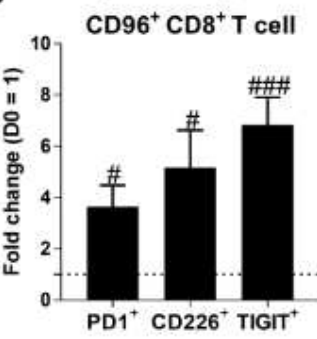

\section{SUPPLEMENTARY FIGURE 4}

a
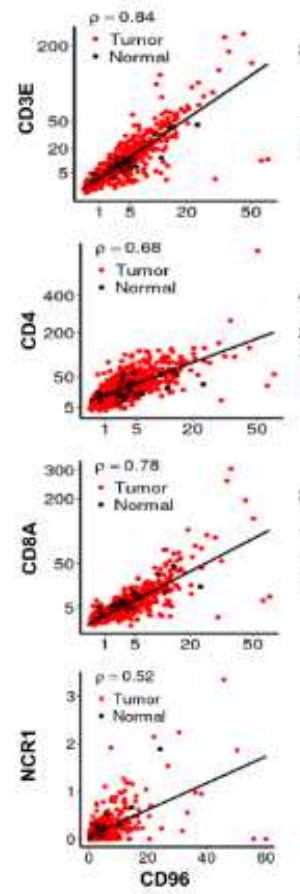
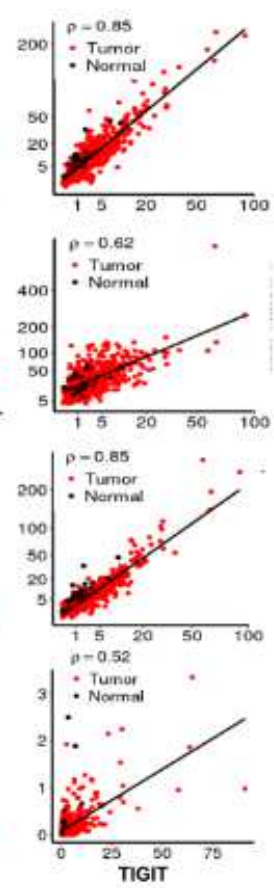

b
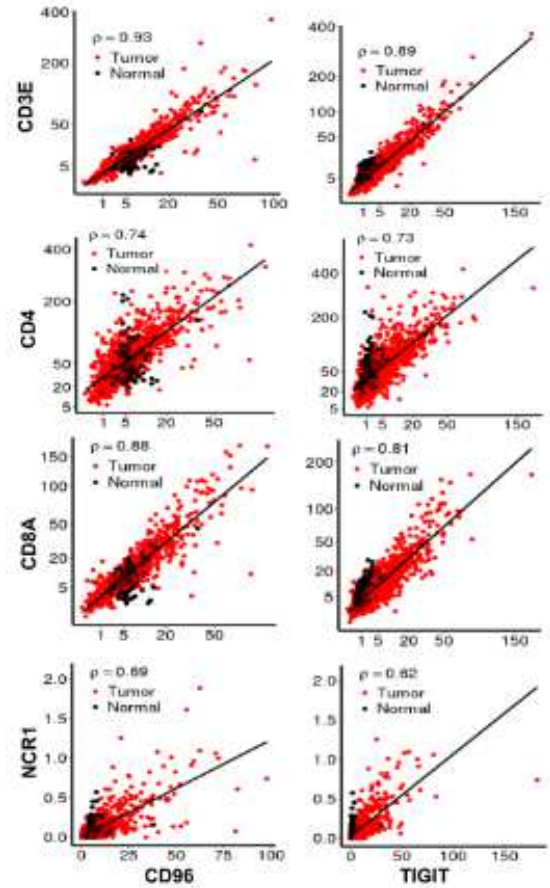
SUPPLEMENTARY TABLE 1

\begin{tabular}{|c|c|c|c|}
\hline Donor & Tetramer & $\begin{array}{c}\text { Tetramer } \\
\text { frequency }\end{array}$ & $\begin{array}{c}\text { Tetramer+ } \\
\text { CD96 } \\
\text { percentagh }\end{array}$ \\
\hline Donor1 & B8-ELK & 7.38 & 14.5 \\
\hline Donor1 & B8-FLR & 0.97 & 24.74 \\
\hline Donor1 & B8-RAK & 1.6 & 18.13 \\
\hline Donor1 & A1-VTE & 1.38 & 19.57 \\
\hline Donor2 & A1-VTE & 2.89 & 23.18 \\
\hline Donor2 & B8-ELK & 0.089 & 29.21 \\
\hline Donor2 & B8-RAK & 0.184 & 34.78 \\
\hline Donor2 & B7-RPH & 0.78 & 23.08 \\
\hline Donor3 & B35-HPV & 3.09 & 59.87 \\
\hline Donor3 & A2-NLV & 0.98 & 50.00 \\
\hline Donor4 & A1-VTE & 3.63 & 9.92 \\
\hline Donor4 & B8-ELK & 21.84 & 13.00 \\
\hline
\end{tabular}




\section{SUPPLEMENTARY TABLE 2}

CyTOF mAbs

\begin{tabular}{|c|c|c|c|c|}
\hline Test & Antigen & Fluorochrome & Clone & Supplier \\
\hline T cells & & & & \\
\hline & CD45 & Pd104 & HI30 & BD \\
\hline & CD45 & Pd110 & HI30 & BD \\
\hline & CD19 & Nd142 & HIB19 & Biolegend \\
\hline & CD8A & Nd145 & RPA-T8 & Biolegend \\
\hline & CD3 & Gd154 & UCHT1 & BD \\
\hline & CD96 & Yb173 & 6F9 & BD \\
\hline & CD4 & Yb174 & SK3 & Fluidigm \\
\hline & FoxP3 & Dy162 & PCH01 & eBioscience \\
\hline & Cisplatin & Pt194/195 & NA & Fluidigm \\
\hline
\end{tabular}

Flow cytometry mAbs

\begin{tabular}{|c|c|c|c|c|}
\hline Test & Antipen & Fluorocturome & Clone & Supplier \\
\hline \multicolumn{5}{|c|}{ Narve, EM, EM RA, CM } \\
\hline & CD4 & FITC & RPA-T4 & BD \\
\hline & CDB & $A P C-C y 7$ & SK1 & BD \\
\hline & $\mathrm{CD}_{3}$ & BV786 & SKT & BD \\
\hline & CD45RA & PB & $\mathrm{H} 1100$ & Biolegend \\
\hline & CCR7 & PE-Cy 7 & $3 \mathrm{D} 12$ & BD \\
\hline & CD96 & PE & NK92 39 & eBioscience \\
\hline & TIGIT & APC & $\mathrm{MBSA} 43$ & eBioscience \\
\hline & CD226 & BV711 & DX11 & BD \\
\hline & PD-1 & BV605 & EH I2.1 & BD \\
\hline \multicolumn{5}{|c|}{$\gamma \overline{0} \mathrm{~T}$ cells and MAIT cells } \\
\hline & CD3: & AF700 & UCHT1 & eBioscience \\
\hline & TCR fo $^{\circ}$ & BV421 & B1 & BD \\
\hline & TCR va7 2 & FITC & $3 \mathrm{ClO}$ & Biolegend \\
\hline & CD161 & PerCP-Cy 5.5 & HP-3G10 & Biolegend \\
\hline & CD96 & $\mathrm{PE}$ & NK92.39 & eBioscience \\
\hline & TIGIT & $\mathrm{APC}$ & MBSA43: & eBioscience \\
\hline & CD226 & BV711 & DX11 & BD \\
\hline & PD-1 & BV605 & $\mathrm{EH} 12.1$ & $\mathrm{BD}$ \\
\hline \multicolumn{5}{|c|}{ Th1. Th2 and Th17 } \\
\hline & CD3 3 & APC-fluor780 & SK 7 & eBioscience \\
\hline & $\mathrm{CD4}$ & BV786 & SK.3 & BD \\
\hline & II. 17-A & BV421 & $\mathrm{N} 49-653$ & BD \\
\hline & INFY & $A F 700$ & $\mathrm{~B} 27$ & BD \\
\hline & II 4 & $\mathrm{PE}-\mathrm{Cy} 7$ & 8D4-8 & BD \\
\hline & CD96 & PE & NK92.39 & eBioscience \\
\hline & TIGIT & A.PC & MBSA43 & eBioscience \\
\hline & $\mathrm{CD}_{226}$ & BV711 & DX11 & BD \\
\hline & PD-1 & BV605 & EH12.1 & BD \\
\hline \multicolumn{5}{|l|}{ Treg } \\
\hline & CD3 & APC-fluor 780 & SK7 & eBioscience \\
\hline & CD4 & AF700 & RPA-T4 & Biolegend \\
\hline & CD25 & PECy 7 & $\mathrm{BC96}$ & Biolegend \\
\hline & FoxP3 & APC & PCH101 & eBioscience \\
\hline & CD127 & BV786 & HIL-7R-M21 & BD \\
\hline & CD96 & $\mathrm{PE}$ & NK92.39 & eBioscience \\
\hline & THGIT & PerCP-eFluor710 & MBSA43 & eBioscience \\
\hline & CD226 & BV711 & DX11 & BD \\
\hline & PD-1 & BV605 & $\mathrm{EH} 12.1$ & $\mathrm{BD}$ \\
\hline \multicolumn{5}{|c|}{ I cells, NKT, NK, myeloid and B cells } \\
\hline & CD3 & BV786 & SKT & BD \\
\hline & CD56 & PE-Cy 7 & B159 & $\mathrm{BD}$ \\
\hline & CD16 & AF700 & $3 \mathrm{G} 8$ & Biolegend \\
\hline & CD19 & BV421 & HIB 19 & BD \\
\hline & CD14 & $\mathrm{APC}-\mathrm{Cy} 7$ & MSE2 & Biolegend \\
\hline & CD155 & PerCP-Cy5.5 & SKII.4 & Biolegend \\
\hline & CD96 & PE & NK92.39 & eBioscience \\
\hline & TIGIT & APC & MBSA43 & eBioscience \\
\hline & CD226 & BV711 & DXII & BD \\
\hline & PD-1 & BV605 & $\mathrm{EH} 12.1$ & $\mathrm{BD}$ \\
\hline
\end{tabular}




\section{SUPPLEMENTARY TABLE 3}

Upregulated in $\mathrm{CD}^{+} \mathrm{T}$-cells

\begin{tabular}{|c|c|c|c|}
\hline Gene & P value & $\begin{array}{c}\text { Mean CD96 } 6^{\text {Low }} \\
(\log 2)\end{array}$ & $\begin{array}{c}\text { Mean CD } 96^{\text {High }} \\
(\log 2)\end{array}$ \\
\hline KLF2 & 0 & 9.399 & 9.075 \\
\hline GZMB & o & 7.95 & 6.088 \\
\hline CCL 4 & 0 & 10.634 & 9.45 \\
\hline TBX21 & 0.0002 & 8.626 & 8.233 \\
\hline IFNG & 0.0003 & 9.249 & 8.346 \\
\hline CTLA4 & 0.0004 & 5.003 & 4.493 \\
\hline CD38 & 0.0004 & 3.676 & 2.078 \\
\hline GZMH & 0.0006 & 6.839 & 5.106 \\
\hline KLRG1 & 0.0007 & 7.3 & 6.735 \\
\hline CD300A & 0.0008 & 4.802 & 3.7 \\
\hline TNF & 0.002 & 9.948 & 9.346 \\
\hline FASLG & 0.0026 & 6.56 & 6.023 \\
\hline GZMM & 0.0029 & 6.517 & 6.052 \\
\hline EGR2 & 0.0037 & 5.836 & 5.316 \\
\hline PVRIG & 0.0042 & 6.289 & 5.834 \\
\hline GZMA & 0.0049 & 7.228 & 6.718 \\
\hline JUNB & 0.0052 & 9.229 & 8.845 \\
\hline RUNX3 & 0.006 & 10.105 & 9.939 \\
\hline PRDM1 & 0.0061 & 7.3 & 6.846 \\
\hline IRF4 & 0.01 & 7.848 & 7.463 \\
\hline NFATC2 & 0.013 & 9.626 & 9.418 \\
\hline CD 8A & 0.018 & 10.918 & 10.79 \\
\hline IL 1ORA & 0.021 & 4.854 & 4.58 \\
\hline CD8B & 0.024 & 7.529 & 7.181 \\
\hline HLA.DRA & 0.025 & 6.71 & 6.122 \\
\hline CCL 5 & 0.026 & 11.156 & 10.946 \\
\hline BCL 6 & 0.027 & 7.809 & 7.655 \\
\hline SOCS3 & 0.031 & 7.277 & 7.06 \\
\hline JUN & 0.036 & 10.763 & 10.625 \\
\hline LAG3 & 0.039 & 6.327 & 6.022 \\
\hline JAK3 & 0.046 & 6.148 & 5.942 \\
\hline
\end{tabular}

Downregulated in CD8 ${ }^{+} \mathrm{T}$-cells

\begin{tabular}{|c|c|c|c|}
\hline Gene & P value & $\begin{array}{c}\text { Mean CD } 96^{\text {Low }} \\
(\log 2)\end{array}$ & $\begin{array}{c}\text { Mean CD } 96^{\text {High }} \\
(\log 2)\end{array}$ \\
\hline GATA3 & 0.0001 & 8.765 & 9.064 \\
\hline ITGAE & 0.0002 & 5.838 & 6.557 \\
\hline CD3D & 0.0003 & 10.374 & 10.561 \\
\hline CCR9 & 0.0004 & 1.51 & 4.349 \\
\hline AHR & 0.0005 & 9.805 & 10.193 \\
\hline IL 7R & 0.0006 & 10.251 & 10.743 \\
\hline NT5E & 0.0014 & 3.476 & 5.16 \\
\hline IL 2RA & 0.0016 & 3.329 & 4.923 \\
\hline CCR6 & 0.0016 & 6.072 & 7.196 \\
\hline CD96 & 0.0024 & 9.085 & 9.401 \\
\hline IL32 & 0.0032 & 8.285 & 8.531 \\
\hline CCR7 & 0.0058 & 7.907 & 8.328 \\
\hline IL 4R & 0.0061 & 8.686 & 9.093 \\
\hline NFKB 1 & 0.009 & 9.089 & 9.278 \\
\hline $\mathrm{BACH} 2$ & 0.01 & 8.015 & 8.396 \\
\hline IL 27RA & 0.013 & 7.689 & 7.86 \\
\hline TNFRSF 18 & 0.016 & 2.671 & 3.682 \\
\hline KLRB 1 & 0.016 & 10.332 & 10.825 \\
\hline STAT4 & 0.018 & 9.59 & 9.705 \\
\hline CXCR6 & 0.033 & 7.107 & 7.532 \\
\hline CD28 & 0.033 & 7.273 & 7.44 \\
\hline IL 12 RB 2 & 0.046 & 4.033 & 4.768 \\
\hline ZBTB 16 & 0.05 & 5.719 & 6.35 \\
\hline
\end{tabular}




\section{SUPPLEMENTARY TABLE 4}

Upregulated in CD4+ T-cells

\begin{tabular}{|c|c|c|c|}
\hline Gene & $\mathrm{P}$ value & $\begin{array}{c}\text { Mean CD96 } \\
(\log 2)\end{array}$ & $\begin{array}{c}\text { Mean CD96 } 6^{\text {High }} \\
(\log 2)\end{array}$ \\
\hline JUNB & 0 & 9.738 & 9.002 \\
\hline IRF4 & 0 & 7.966 & 6.957 \\
\hline CTLA4 & 0 & 7.389 & 6.166 \\
\hline SOCS3 & 0.0001 & 8.232 & 7.56 \\
\hline KLF2 & 0.0001 & 9.801 & 9.067 \\
\hline JAK3 & 0.0001 & 7.248 & 6.569 \\
\hline CXCR5 & 0.0001 & 6.098 & 3.274 \\
\hline ICOS & 0.0002 & 9.863 & 9.217 \\
\hline SELL & 0.0003 & 7.136 & 5.734 \\
\hline PVRIG & 0.0003 & 6.105 & 5.501 \\
\hline FAS & 0.0007 & 8.65 & 8.347 \\
\hline CD45RA & 0.0014 & 9.188 & 8.735 \\
\hline CCL4 & 0.0019 & 7.703 & 6.858 \\
\hline IFNG & 0.0023 & 7.89 & 7.353 \\
\hline STAT5B & 0.0035 & 8.978 & 8.725 \\
\hline CCR7 & 0.0038 & 9.087 & 8.653 \\
\hline CD27 & 0.0039 & 6.516 & 5.654 \\
\hline TIGIT & 0.004 & 7.407 & 6.053 \\
\hline FOXP3 & 0.0045 & 5.565 & 3.507 \\
\hline JAK1 & 0.0047 & 9.958 & 9.806 \\
\hline JUN & 0.0049 & 10.709 & 10.464 \\
\hline STAT1 & 0.0054 & 8.839 & 8.489 \\
\hline STAT5A & 0.0055 & 9.359 & 9.106 \\
\hline CD69 & 0.0057 & 10.9 & 10.583 \\
\hline SOCS1 & 0.0093 & 7.92 & 7.648 \\
\hline GZMB & 0.02 & 4.218 & 3.229 \\
\hline HLA.DRA & 0.028 & 6.501 & 5.812 \\
\hline CD28 & 0.028 & 8.652 & 8.412 \\
\hline STAT3 & 0.038 & 9.812 & 9.596 \\
\hline MAF & 0.038 & 8.614 & 8.392 \\
\hline HAVCR2 & 0.046 & 4.25 & 3.753 \\
\hline CD226 & 0.048 & 6.694 & 6.558 \\
\hline
\end{tabular}

Downregulated in CD4+ T-cells

\begin{tabular}{|c|c|c|c|}
\hline Gene & P value & $\begin{array}{c}\text { Mean CD96 } \\
(\log 2)\end{array}$ & $\begin{array}{c}\text { Mean CD96 } \\
(\log 2)\end{array}$ \\
\hline CD96 & 0 & 8.951 & 9.347 \\
\hline CCR6 & 0 & 7.357 & 8.263 \\
\hline LGALS1 & 0.0001 & 7.555 & 8.24 \\
\hline STAT4 & 0.0014 & 9.142 & 9.358 \\
\hline AHR & 0.0041 & 10.814 & 11.13 \\
\hline NT5E & 0.0044 & 2.801 & 3.738 \\
\hline CXCR3 & 0.0049 & 7.993 & 8.33 \\
\hline RORC & 0.0071 & 4.5 & 5.534 \\
\hline IL32 & 0.01 & 8.659 & 8.842 \\
\hline JAK2 & 0.014 & 6.591 & 6.828 \\
\hline EGR2 & 0.026 & 5.147 & 5.592 \\
\hline TGFB1 & 0.032 & 11.717 & 11.809 \\
\hline CXCR6 & 0.032 & 5.991 & 6.621 \\
\hline IFNGR1 & 0.044 & 8.929 & 9.148 \\
\hline
\end{tabular}




\section{SUPPLEMENTARY TABLE 5}

Differentially expressed genes in activated CD96 ${ }^{\text {high }}$ and CD96 low T-cells

\begin{tabular}{|c|c|c|c|}
\hline Gene & P value & $\begin{array}{c}\text { Mean CD96 } \\
(\log 2)\end{array}$ & $\begin{array}{c}\text { Mean CD96 } \\
(\log 2)\end{array}$ \\
\hline ICOS & 0.0006 & 6.5920 & 5.9760 \\
\hline IL2 & 0.0023 & 11.3260 & 12.5860 \\
\hline NT5E & 0.047 & 5.3900 & 6.2540 \\
\hline CD160 & 0.0061 & 6.9280 & 6.3920 \\
\hline IL23A & 0.012 & 4.7640 & 5.6580 \\
\hline GZMB & 0.012 & 9.0460 & 8.3820 \\
\hline CD45RA & 0.012 & 6.5040 & 6.1260 \\
\hline JAK1 & 0.012 & 5.5920 & 4.9660 \\
\hline ITGA4 & 0.02 & 4.0400 & 3.2840 \\
\hline IL17A & 0.02 & 2.8340 & 4.7440 \\
\hline IL22 & 0.028 & 2.5200 & 4.6780 \\
\hline STAT6 & 0.033 & 4.8660 & 4.5520 \\
\hline JUNB & 0.036 & 4.8200 & 4.5520 \\
\hline STAT5B & 0.038 & 6.0420 & 5.6000 \\
\hline
\end{tabular}

Genes differentially expressed comparing fold change of CD96 high and CD96low T-cells following activation

\begin{tabular}{|c|c|c|c|}
\hline Gene & P Value & $\begin{array}{c}\text { Mean FC CD96 } \\
(\log 2)\end{array}$ & $\begin{array}{c}\text { Mean FC } \\
\text { CD96 } 6^{\text {High }}(\log 2)\end{array}$ \\
\hline CCL4 & 0.014 & 7.9500 & 7.0509 \\
\hline IFNG & 0.04 & 8.7258 & 8.0529 \\
\hline IL23A & 0.019 & 1.7574 & 1.0766 \\
\hline GZMM & 0.012 & -0.9019 & -2.4781 \\
\hline CD3D & 0.045 & -0.3885 & -0.1079 \\
\hline CD45RA & 0.001 & -1.5519 & -1.0846 \\
\hline CD69 & 0.032 & 0.1565 & 0.3086 \\
\hline CD244 & 0.047 & -1.1670 & 0.0254 \\
\hline CTLA4 & 0.037 & -1.0291 & -2.5901 \\
\hline FOS & 0.028 & -2.9770 & -2.6471 \\
\hline NFKB1 & 0.016 & 0.7128 & 0.8980 \\
\hline JAK1 & 0.014 & -2.2049 & -1.4512 \\
\hline SOCS5 & 0.016 & -1.3261 & -2.4337 \\
\hline
\end{tabular}

